\title{
Le tritium organique dans les écosystèmes d'eau douce : évolution à long terme dans l'environnement des centres nucléaires de production d'électricité français
}

\author{
G. GONTIER ${ }^{1}$, F. SICLET ${ }^{2}$
}

(Manuscrit reçu le 4 janvier 2011, accepté le 18 juin 2011)

RÉSUMÉ

De 1977 à 2009, plus de 600 analyses d'activités en tritium organique ont été réalisées sur les poissons, les végétaux aquatiques et les sédiments, en amont et en aval des 15 CNPE français situés en bord de rivière. L'exploitation de ces résultats montre que les activités en tritium organique ont diminué de façon exponentielle au cours des trente dernières années, dans tous les compartiments des écosystèmes aquatiques. Dans les zones en amont de tout CNPE, les activités en tritium organique dans les sédiments sont supérieures aux activités mesurées dans les poissons et les végétaux, elles-mêmes supérieures aux activités en HTO des eaux de surface. L'ampleur de ces écarts dépend du bassin concerné et s'explique par la nature différente des sources de tritium. Dans les bassins versants où les retombées des essais nucléaires atmosphériques constitue la source principale de tritium, les niveaux observés résultent de l'exposition des organismes aquatiques à deux formes distinctes de tritium d'âges différents : l'eau tritiée de l'atmosphère, image des retombées au moment du prélèvement, et le tritium organique des sols, formé sur plusieurs dizaines d'années, qui alimente le compartiment sédimentaire des cours d'eau. Dans les bassins du Rhône et du Rhin, une source supplémentaire de tritium de très faible biodisponibilité, provenant vraisemblablement de l'industrie des peintures luminescentes, marque la matière organique des sédiments à hauteur de 100 à $100000 \mathrm{~Bq} . \mathrm{L}^{-1}$ d'eau de combustion. La comparaison des niveaux observés en amont et en aval des CNPE montre que l'influence des rejets est détectable seulement dans les cours d'eau où le bruit de fond est faible, c'est-à-dire dans les bassins autres que ceux du Rhône et du Rhin. L'augmentation des activités en tritium organique dans les végétaux et les poissons est inférieure à l'augmentation de l'activité en HTO due aux rejets, ce qui témoigne, d'une part, de l'absence de phénomène de bioaccumulation à partir de l'eau tritiée et, d'autre part, de l'absence de composés organiques bioaccumulables dans les rejets.

ABSTRACT Organic tritium in freshwater ecosystems: long-term trends in the environment of French nuclear power plants.

From 1977 to 2009, more than 600 measurements of organic tritium were performed on fish, aquatic plants and sediments upstream and downstream of the 15 French NPP located along rivers. Examination of the results shows that organic tritium activities have exponentially decreased over the last thirty years, in all components

\footnotetext{
IRSN, DEI, BP 3, 13115 Saint-Paul-Lez-Durance, France.

Adresse actuelle : gilles.gontier@edf.fr

2 EDF R\&D, LNHE, 6 quai Watier, 78400 Chatou, France.
} 


\begin{abstract}
of aquatic ecosystems. Upstream of all NPP, OBT levels in sediments are higher than in plants and fish, themselves larger than HTO in surface water. The magnitude of these differences and the long-term trends depend on the river basin and can be explained by the varying nature of tritium sources. In river catchments, where atmospheric test fallout is the main source of tritium, the observed levels result from the exposure of aquatic organisms to two distinct tritium pools of different ages : atmospheric tritiated water (representing present fallout), and organic tritium from soils (formed over several decades) which supplies particulate matter to surface waters. In the Rhône and Rhine river basins, an additional source of organic tritium of very low bio-availability, probably originating from the luminescent paint industry, is responsible for the spiking of sediment organic matter up to 100 to $100000 \mathrm{~Bq} . \mathrm{L}^{-1}$ combustion water. The comparison of upstream and downstream NPP tritium levels shows that the influence of tritium discharges is detectable only in rivers, with low background OBT activities, i.e in basins other than the Rhône and Rhine. The observed increase in plant and fish OBT is lower than the added HTO activity in water due to discharge, which supports the absence of bioaccumulation for tritium originating from HTO and the absence of highly bio-available tritiated organic molecules in NPP discharges.
\end{abstract}

Keywords: Organically bound tritium / freshwater/ aquatic plant / sediment / fish / bioaccumulation

\title{
1. Introduction
}

Le tritium, isotope radioactif de l'hydrogène, dont la demi-vie est de 12,32 ans, fait partie des radionucléides majoritaires en terme d'activité dans les rejets d'effluents des CNPE (Centres nucléaires de production d'électricité). En l'absence de méthodes de traitement, de piégeage et/ou de stockage applicable aux systèmes industriels, et parce que sa radiotoxicité est faible, ce radionucléide est rejeté dans le milieu récepteur sous forme gazeuse ou liquide dans le respect de la réglementation en vigueur. Dans un contexte d'augmentation des rejets de tritium liée à la construction de nouveaux réacteurs électro-nucléaires et à l'installation de recherche ITER, le questionnement sur l'impact environnemental et sanitaire du tritium est d'actualité, aussi bien chez le législateur et dans les milieux scientifiques, qu'auprès du public et des associations de protection de l'environnement. Un des points qui fait plus particulièrement l'objet de débat concerne la bioaccumulation du tritium dans les chaînes alimentaires, notamment suite aux mesures dans l'eau et les poissons de la baie de Bristol (McCubbin et al., 2001) qui contrediraient l'hypothèse d'équilibre isotopique entre le tritium rejeté sous forme d'eau tritiée et le tritium lié à la matière organique vivante.

Sur le territoire français, de nombreuses analyses d'activités en tritium organique ont été réalisées au cours des trois dernières décennies en lien avec les suivis radioécologiques des sites où des CNPE sont exploités. Ces données qui pour la plupart n'ont été publiées que dans les rapports de surveillance de ces ouvrages sont rassemblées ici pour ce qui concerne le milieu aquatique continental. Le but de cet article est d'exploiter ces résultats pour présenter une vue d'ensemble des niveaux en tritium organique des principaux bassins hydrographiques français, d'analyser 
les tendances d'évolution temporelle et de caractériser l'influence des rejets d'effluents des CNPE, dans le cadre de la réflexion sur le comportement du tritium organique dans les écosystèmes aquatiques. Les connaissances existantes sur le tritium organique dans l'environnement ayant fait l'objet de nombreuses synthèses (Blaylock et al., 1986; Diabate et Strack, 1993; Belot et al., 1996; Boyer et al., 2009), cet article ne fait pas une revue exhaustive sur ce thème mais s' attache plutôt à souligner les mécanismes importants pour lesquels les mesures de surveillance apportent de nouveaux éléments de compréhension et de quantification.

\section{Formes du tritium}

Dans l'environnement, la plus grande partie du tritium, d'origine naturelle ou anthropique, se trouve sous forme d'eau tritiée (HTO) à l'état liquide ou gazeux. En dehors des zones de rejets industriels, les précipitations constituent la voie d'entrée principale de l'eau tritiée dans l'hydrosphère. La circulation de l'eau tritiée suit celle de l'eau dans les eaux de surface et les eaux souterraines. Le tritium présent dans ces masses d'eau peut d'ailleurs servir de traceur en hydrologie et en hydrogéologie, notamment pour déterminer la vulnérabilité des aquifères à différentes pollutions ou connaître les temps de séjour moyens des eaux d'un aquifère. L'eau tritiée est rapidement incorporée à l'eau des tissus des êtres vivants qui représente 80 à $95 \%$ de leur masse. Dans les végétaux aquatiques et terrestres, le tritium entre dans le cycle de la matière organique par la photosynthèse à partir de l'eau tritiée. Ce tritium appelé tritium organique (en abrégé, TOL en français, pour Tritium Organiquement Lié, et OBT en anglais, pour Organically Bound Tritium) est ensuite utilisé, transformé, dégradé, le long des chaînes trophiques. L'eau tritiée et le tritium organique, en raison de leur forme chimique très différente ont des comportements très différents et donc des répartitions très différentes dans l'environnement. Par exemple, le temps de séjour de l'eau tritiée dans le cours d'eau, en un lieu donné, est très court alors que le tritium une fois incorporé à la matière organique peut, selon la nature des composés formés, disparaître très lentement (au bout de plusieurs dizaines voire plusieurs centaines d'années pour les arbres). Cette rémanence du tritium organique permet d'utiliser la mesure du TOL des végétaux et des animaux afin d'avoir une information rétrospective sur l'activité moyenne de l'eau tritiée ou de la vapeur d'eau tritiée sur toute la période de formation de la matière organique, alors que la mesure du tritium libre fournit une valeur ponctuelle, image de l'activité volumique dans l'eau ou dans l'air, au moment du prélèvement. Ceci explique la grande variabilité des rapports TOL/ HTO observés in situ. Ainsi, dans le cas des CNPE français dont les effluents radioactifs liquides sont rejetés de façon discontinue, ce rapport peut, pour un même échantillon prélevé à quelques jours d'intervalle, varier de plusieurs ordres de grandeur entre un prélèvement qui 
a lieu en dehors d'une période de rejet, c'est-à-dire lorsque l'activité en tritium sous forme HTO est proche du bruit de fond (activité en HTO inférieure à $1 \mathrm{~Bq} . \mathrm{L}^{-1}$ ), et un prélèvement en période de rejet (activité en HTO pouvant atteindre $70 \mathrm{~Bq} \cdot \mathrm{L}^{-1}$ ).

La matière organique tritiée d'origine végétale et animale se retrouve au final dans la matière organique des sédiments qui est constituée à la fois d'organismes vivants (végétaux, animaux, microbes) et de leurs produits de décomposition. Ce compartiment présente un spectre global de toutes les sources de matière organique du bassin versant, d'origine terrestre (produits de décomposition de la végétation terrestre et lessivage des sols) ou aquatique (organismes benthiques, produits de décomposition des végétaux et animaux aquatiques) auxquels s'ajoutent la matière organique d'origine anthropique provenant des stations d'épuration. La matière organique d'origine terrestre contient notamment les molécules de décomposition de la structure ligno-cellulosique végétale (substances humiques) beaucoup moins biodégradables que celles issues du phytoplancton et autres organismes aquatiques. Il en résulte que la matière organique des sédiments est souvent dominée dans les cours d'eau étudiés par la matière organique particulaire d'origine terrestre, aux caractéristiques proches de celles des sols et litières du bassin versant (Abril et al., 2002 ; Fontugne et al., 2002).

\section{Sites d'études et méthodes d'analyse}

Les premières mesures de tritium lié à la matière organique dans le milieu aquatique autour des CNPE français remontent à 1977. À partir de 1980, lors des points zéro radioécologiques, qui visent à caractériser l'état de l'environnement, avant le démarrage des réacteurs électronucléaires, et lors des bilans radioécologiques décennaux, effectués 10 ans après, le tritium organique est régulièrement analysé dans les poissons. Progressivement, des mesures de tritium organique sur les végétaux aquatiques puis sur les sédiments sont introduites dans ces programmes. Au total, ce sont plus de 600 analyses qui ont été réalisées dans les écosystèmes d'eau douce sur les poissons, les végétaux et les sédiments depuis 1977, autour des 14 sites de centrales REP (réacteurs à eau pressurisée) et du site RNR (réacteur à neutrons rapides) de Creys-Malville (Fig. 1). Pour chaque CNPE, on distingue d'une manière générale trois zones de prélèvement : l'amont de l'installation (à quelques kilomètres en amont), l'aval proche de l'ouvrage de rejet (à quelques kilomètres en aval), l'aval lointain de l'installation (au delà de $10 \mathrm{~km}$ ). L'emplacement exact des stations de prélèvement figure dans les études radioécologiques décennales des CNPE (Calmon, 2011 ; Antonelli, 2007, 2008, 2009, 2010 ; Parache, 2009, 2010a, 2010b ; Gontier, 2004, 2006 ; Duffa, 2003 ; Leprieur et Gontier, 2001 ; Descamps et al., 2000 ; Leprieur, 1999 ; Beaugelin et al., 1997). 


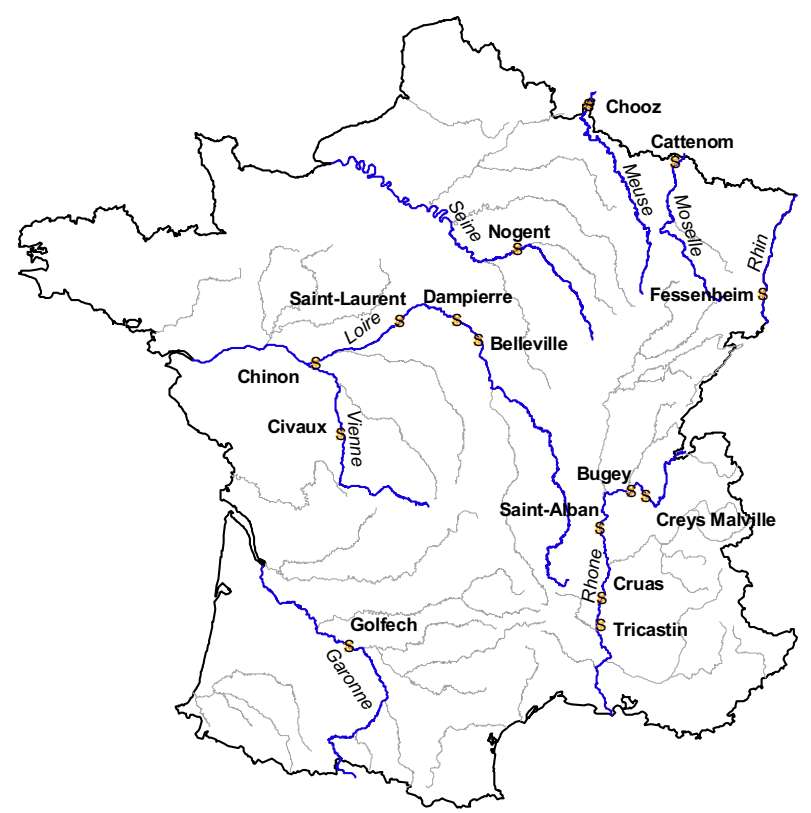

Figure 1 - Localisation des sites étudiés. Les noms des CNPE sont en gras, les noms des cours d'eau sont en italiques.

Location of studied sites - Names of NPP in bold, name of rivers in italics.

\section{Méthodes d'analyses}

La capacité du tritium sous forme d'eau tritiée à s'échanger rapidement entre tous les compartiments du milieu contenant de l'eau est une difficulté majeure de la mesure de ce radionucléide. Ceci s'applique en premier lieu à l'eau libre des échantillons frais avec des possibilités de contamination croisée ou d'échange avec l'air à toutes les étapes entre le prélèvement et la mesure. La difficulté à maintenir un isolement absolu de l'échantillon a conduit à supprimer la mesure du tritium dans l'eau libre des échantillons frais, lors des suivis radioécologiques. Il est préférable, si l'on cherche à avoir une mesure du tritium libre représentative du lieu de prélèvement, de collecter l'eau du système aquatique considéré que l'on peut plus facilement isoler de tout échange avec le milieu ambiant. Cette difficulté de confinement concerne aussi la mesure du tritium échangeable (TOL-E), lié à la matière organique par des groupements $\mathrm{S}-\mathrm{H}, \mathrm{O}-\mathrm{H}$ ou $\mathrm{N}-\mathrm{H}$, qui se met à l'équilibre avec l'eau libre de l'échantillon frais ou avec la vapeur d'eau atmosphérique lorsque l'échantillon est déshydraté, avec les problèmes de contamination que cela peut entraîner. À l'inverse, le tritium lié aux atomes de carbone par des liaisons 
covalentes, que l'on appelle tritium organique non échangeable (TOL-NE), est beaucoup plus stable. On pourrait en déduire que la mesure de cette fraction du tritium n'est pas affectée par les problèmes d'échange que nous venons d'évoquer. Pourtant, comme les techniques de mesures ne permettent pas d'accéder exclusivement au tritium associé aux atomes de carbone, la fraction soumise à la mesure comporte obligatoirement en plus de l'hydrogène et du tritium non échangeables, une part d'hydrogène et de tritium échangeables avec les difficultés de caractérisation propres à cette forme.

Le résultat des analyses de tritium organique est donc fortement dépendant de la méthode de préparation des échantillons et de la technique de mesures (Belot et al., 1996). Il convient d'en tenir compte dans l'interprétation des résultats et les comparaisons entre échantillons. De plus, les protocoles de préparation des échantillons et les techniques de mesures, utilisés dans les suivis radioécologiques des CNPE, ont varié au cours du temps :

- déshydratation par séchage à l'étuve ou lyophilisation,

- étape additionnelle d'élimination du tritium échangeable,

- combustion des échantillons selon différents protocoles (température et durée) puis mesures par scintillation liquide sur eau de combustion,

- ou mesures de l'hélium 3, formé par décroissance du tritium, au moyen d'un spectromètre de masse, sur échantillons déshydratés par lyophilisation.

Nous décrivons ci-après chronologiquement les principales caractéristiques des protocoles utilisés.

Les premières mesures de TOL dans les années 70 à 80 (Foulquier et Pally, 1982), sont effectuées, par le laboratoire de Radiotoxicologie du CEA de Fontenay-aux Roses, sur les échantillons déshydratés à l'étuve puis soumis à une combustion dans un four tubulaire catalytique où la matière organique est oxydée (Jeanmaire et al., 1973). L'eau de combustion est récupérée et distillée sous vide avant d'être mesurée par scintillation liquide. Il n'y a pas d'élimination du tritium échangeable. La mesure correspond donc au tritium organique total (échangeable et non échangeable). D'après le protocole, on peut supposer que le tritium échangeable est à l'équilibre avec la vapeur d'eau du lieu de stockage et de préparation des échantillons.

À partir de 1984, les échantillons sont mesurés au laboratoire IPSN de Pierrelatte. La lyophilisation remplace la déshydratation à l'étuve pour éviter le fractionnement isotopique. Le tritium organique échangeable est éliminé par échange isotopique avec un flux d'air humide non tritié, pendant une semaine à $65{ }^{\circ} \mathrm{C}$ (Guénot, 1984). Les échantillons restent ensuite à l'étuve à $60^{\circ} \mathrm{C}$ jusqu' au passage au four. Tout contact avec l'air du laboratoire est évité. La combustion 
s'opère sous flux d'oxygène avec une montée en température progressive jusqu'à $900{ }^{\circ} \mathrm{C}$ (Camus et al., 1987). L'eau de combustion est récupérée et mesurée par scintillation liquide. La mesure correspond donc au tritium non échangeable lié à la matière organique. Il n'y pas de correction du résultat de mesure pour tenir compte de la dilution par l'eau dépourvue de tritium.

En 1994, le laboratoire de mesure du tritium est transféré de Pierrelatte à Orsay. Les échantillons du bilan décennal aquatique et terrestre de Cruas et ceux du bilan décennal aquatique de Saint-Laurent prélevés en 1993 sont les derniers échantillons mesurés à Pierrelatte. Le transfert du laboratoire a occasionné des retards importants dans les analyses qui ont commencé en 1998 au LMRE d'Orsay, soit après 4 à 5 ans de stockage des échantillons. Ce transfert s'accompagne d'une modification des protocoles de mesures. L'étape d'élimination du tritium échangeable est supprimée, car l'air ambiant dans le laboratoire d'Orsay n'est pas exposé à des augmentations d'activité en tritium qui risqueraient par échange isotopique avec le tritium organique échangeable de l'échantillon de fausser la mesure. En 2001, des résultats aberrants constatés sur des feuilles de chênes amènent à stocker les échantillons en attente de la résolution du problème. Le protocole de combustion est modifié (Cossonnet et al., 2009) et mis en pratique à partir de 2003. Pour résorber le retard, une partie des échantillons, notamment ceux pauvres en matière organique (sols, sédiments) sont confiés au Laboratoire des Sciences du Climat et de l'Environnement de Gif-sur-Yvette (CEA/CNRS) pour une quantification du tritium par la mesure de l'hélium 3 en spectrométrie de masse. On peut donc considérer que les résultats d'analyses en tritium organique sur les échantillons prélevés de 1994 à 2000 ne possèdent pas le même niveau de fiabilité que ceux mesurés à partir de 2001.

En 2002, 2003, et 2005 le LSCE a en charge le suivi radioécologique du site de Creys-Malville (Jean-Baptiste et al., 2007). Le traitement des échantillons comporte les étapes suivantes: lyophilisation, élimination du tritium échangeable par rinçage avec de l'eau considérée comme dépourvue de tritium (activité de $0,19 \mathrm{~Bq} . \mathrm{L}^{-1}$ ) pour MES, sol et sédiment, dosage du tritium par « recroissance » de l'hélium 3 (production de l'hélium 3, descendant stable du tritium, dans un temps connu dans une ampoule scellée), correction du résultat pour tenir compte de la dilution du tritium par rinçage avec l'eau dépourvue de tritium. Pour les échantillons biologiques qui ne sont pas soumis à ce rinçage, l'activité du tritium organique échangeable est considérée égale à celle de l'eau de lyophilisation, la correction pour calculer le tritium organique non échangeable est faite sur cette base.

En 2009, le laboratoire de l'École des Mines de Nantes en charge du suivi radioécologique de Golfech réalise les mesures de tritium organique sur les poissons par scintillation liquide sur échantillon lyophilisé sans élimination du tritium échangeable (SUBATECH, 2010). 


\section{Expression des résultats}

L'unité choisie dans cet article pour exprimer les activités en tritium liée à la matière organique est le becquerel par litre d'eau de combustion. C'est le résultat initial de la mesure du tritium par scintillation liquide après combustion des échantillons secs, c'est aussi l'unité qui permet une comparaison directe entre échantillons quelle que soit leur nature, y compris avec l'activité en tritium dans l'eau. Pour convertir les becquerels par litre d'eau de combustion en becquerel par gramme d'hydrogène, il faut multiplier par 0,009 en se basant sur la masse relative d'hydrogène dans la molécule d'eau. Pour exprimer les résultats de TOL en $\mathrm{Bq} . \mathrm{kg}^{-1} \mathrm{sec}$, il est nécessaire de connaitre la teneur en hydrogène de la matière organique de l'échantillon ou le volume d'eau de combustion rapporté au poids sec (en anglais «water equivalent factor»), variable d'un échantillon à l'autre. Pour les poissons, la valeur moyenne du « water equivalent factor » fournie par l'AIEA (IAEA, 2010) est 0,65 L d'eau de combustion par kg sec. Celle utilisée par le CEA pour l'ensemble des matrices biologiques dans les années 80 est 0,63 (Jeanmaire et al., 1973). Il faut multiplier les résultats en Bq. $\mathrm{L}^{-1}$ d'eau de combustion par ces valeurs pour obtenir une activité en Bq. $\mathrm{kg}^{-1} \mathrm{sec}$. Lorsque le tritium est mesuré par spectrométrie de masse sur l'hélium 3, le résultat initial est exprimé en mole $\mathrm{d}^{3} \mathrm{H}^{3} \cdot \mathrm{kg}^{-1} \mathrm{sec}$. Il est donc nécessaire là aussi de déterminer la teneur en hydrogène de la matière organique de l'échantillon pour convertir le résultat en mole d ${ }^{3} \mathrm{H}$ par mole d' $\mathrm{H}$, puis en Bq. $\mathrm{L}^{-1}$ d'eau de combustion. Les résultats sont parfois exprimés en unités tritium, en abrégé UT ; 1 UT correspond à $10^{-18}$ mole d ${ }^{3} \mathrm{H}$ par mole d'H et $0,118 \mathrm{~Bq} \cdot \mathrm{L}^{-1} \mathrm{~d}$ 'eau de combustion.

En toute rigueur si l'on veut calculer le rapport $\left({ }^{3} \mathrm{H} / \mathrm{H}\right)_{\text {non -ech }}$ entre le tritium non échangeable et l'hydrogène non échangeable, il faut connaître la proportion $\alpha$ d'hydrogène échangeable par rapport à l'hydrogène total présent dans l'échantillon lyophilisé (généralement entre 20 et $30 \%$ pour les tissus végétaux et animaux) et l'activité en tritium échangeable ${ }^{3} \mathrm{H}_{\text {ech }}$ de l'échantillon soumis à la combustion ou à la mesure par spectrométrie de masse. Le rapport $\left({ }^{3} \mathrm{H} / \mathrm{H}\right)_{\text {non -ech }}$ est calculé alors à partir de la formule suivante (Jean-Baptiste et al., 2010) :

$$
\begin{gathered}
\left({ }^{3} \frac{H}{H}\right)_{\text {non-ech }}=\frac{\left[\left(\frac{{ }^{3}}{H}\right)_{\text {eau-combustion }}-\alpha \cdot\left(\frac{{ }^{3}}{H}\right)_{\text {ech }}\right]}{1-\alpha} \\
\text { avec }\left(\frac{{ }^{3}}{H}\right)_{\text {non-ech }},\left({ }^{3} \frac{H}{H}\right)_{\text {eau-combustion }},\left({ }^{3} \frac{H}{H}\right)_{\text {ech }} \text { exprimés en } \quad \mathrm{Bq} \cdot \mathrm{g}^{-1}
\end{gathered}
$$
d'hydrogène. 
Si l'échantillon a subi une étape d'élimination du tritium par rinçage avec une eau dépourvue de tritium, l'activité en tritium échangeable est nulle. La proportion $\alpha$ d'hydrogène échangeable n'étant pas une grandeur connue avec précision pour les différentes natures d'échantillons environnementaux, cela constitue une limitation pour l'évaluation exacte des rapports entre le tritium non échangeable et l'hydrogène non échangeable dans la matière organique.

\section{Incertitudes de mesures}

L'incertitude sur la mesure en spectrométrie de masse de l'hélium 3 est en moyenne de $5 \%$ sur les résultats présentés ici. En scintillation liquide, l'incertitude de comptage est de l'ordre de $10 \%$, avec une variabilité beaucoup plus importante, notamment lorsque les activités sont faibles (l'incertitude peut, dans ce cas, aller jusqu'à $50 \%$ ). Il est clair que l'incertitude de comptage est faible par rapport aux autres sources de variation tout au long de la chaîne de traitement des échantillons, comme le montrent les exercices d'inter-comparaison (Workman et al., 2005; Boyer et al., 2009; Baglan et al., 2010). Dans ce contexte, il convient de souligner que toute interprétation quantitative nécessite de grandes séries de données accompagnées de tests statistiques de significativité.

Deux types de tests ont été utilisés dans ce travail :

- pour tester si la pente des courbes de tendance d'évolution temporelle était significativement différente de zéro, le test de Student (Annexe - Tab. I).

- pour les comparaisons entre séries de données, le test de Student apparié lorsque le jeu de données le permettait, sinon le test non paramétrique de Mann et Whitney (Annexe - Tab. II).

Pour l'ensemble de ces tests, le seuil de $95 \%$ a été choisi comme limite de significativité.

\section{Résultats}

\subsection{Sédiments}

Les 164 mesures de tritium organique (TOL) réalisées dans les sédiments sont représentées sur la figure 2. Aucune analyse sur ce type de matrice n'est inférieure à la limite de détection qui est d'environ $1 \mathrm{~Bq} \cdot \mathrm{L}^{-1}$. Deux groupes se distinguent clairement :

- l'ensemble constitué par le Rhône et le Rhin avec des activités en tritium organique comprises entre 100 et 100000 Bq. $\mathrm{L}^{-1}$ d'eau de combustion,

- les autres cours d'eau avec des activités entre 2 et 100 Bq.L ${ }^{-1}$ d'eau de combustion. 


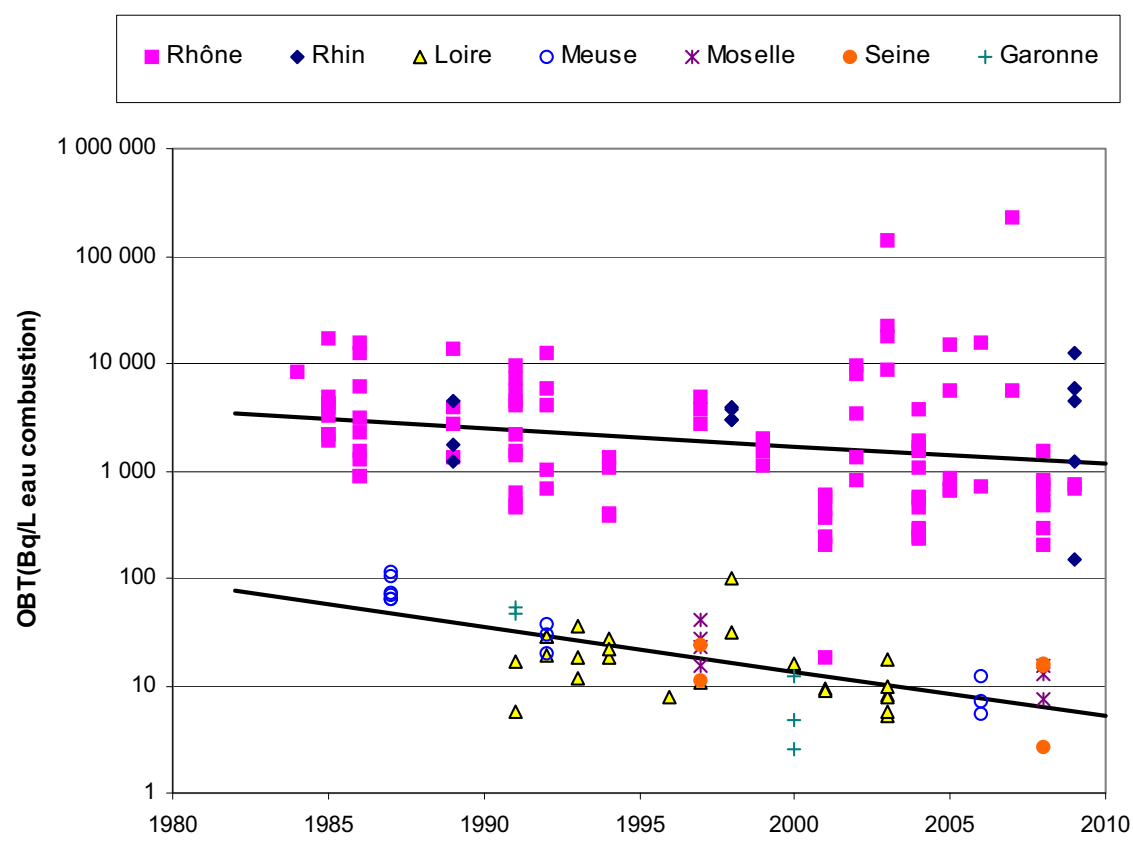

Figure 2 - Évolution des activités en tritium organiquement lié (TOL) dans les sédiments autour des CNPE (toutes stations).

Evolution of organically bound tritium $(O B T)$ in sediments around power plants (all sampling sites).

Dans le premier ensemble, trois valeurs singulières ressortent : deux valeurs beaucoup plus fortes que les autres, observées dans la partie amont du Rhône (226 000 Bq.L ${ }^{-1}$, en 2007 au port de Groslée, $15 \mathrm{~km}$ en amont de tous les CNPE, 136000 Bq.L ${ }^{-1}$ en 2003 au barrage de Villebois, entre Creys et Bugey), et une valeur beaucoup plus faible que les autres $\left(18 \mathrm{~Bq} . \mathrm{L}^{-1}\right)$ en 2001 , à proximité du CNPE de Tricastin. Ce prélèvement n'a pas été fait directement dans le Rhône, mais dans un lac, le Lac Le Trop Long, potentiellement alimenté par la nappe phréatique en aval du CNPE de Tricastin, mais non exposé à des apports sédimentaires venant du Rhône.

À l'échelle temporelle, on constate une tendance à la décroissance des activités en TOL des sédiments selon une régression de type exponentielle, significative au seuil de 99 \% pour l'ensemble Rhône-Rhin, avec une demi-vie apparente ${ }^{3}$ de

\footnotetext{
${ }^{3}$ La demi-vie apparente, ou demi-vie effective, est l'intervalle de temps nécessaire pour passer d'une activité A en tritium dans l'environnement à une activité $\mathrm{A} / 2$. Elle est calculée ici à partir de la pente de décroissance de la droite de régression représentée sur la figure 2, selon la formule : demi-vie $(\mathrm{an})=0,693 /$ pente $\left(\mathrm{an}^{-1}\right),(c f$. Annexe - Tab. I)
} 
17,3 ans, et significative au seuil de 99,99\% pour les autres cours d'eau avec une pente, correspondant à une demi-vie de 7,2 ans (Annexe - Tab. I). Cette tendance est à mettre en relation avec la décroissance du tritium produit en très grandes quantités lors des essais nucléaires atmosphériques des années 60. La demi-vie apparente sur l'ensemble Rhin et Rhône supérieure à la demi-vie radioactive du tritium traduit l'existence d'apport continu de tritium sur ces bassins, alors que sur les autres cours d'eau, le tritium diminue plus rapidement que la décroissance radioactive en raison de la dilution par la matière organique récente moins marquée.

Afin de mieux caractériser les apports des bassins versants, des prélèvements de matières en suspension (MES) ont été réalisés dans des décanteurs lamellaires sur le Rhône, la Seine et la Loire en amont de toutes les centrales, à plusieurs saisons (Fig. 3). Les MES exportées par le bassin versant du Rhône amont entre 2002 et 2004 ont une teneur moyenne en TOL de 13000 Bq.L ${ }^{-1}$ d'eau de combustion (Annexe - Tab. III), témoignant d'un apport continu de matière organique fortement tritiée alors que la Seine et la Loire présentent des activités moyennes de 44 et 19 Bq. $\mathrm{L}^{-1}$ d'eau de combustion respectivement, les différences entre Seine et Loire n'étant pas statistiquement significatives (Annexe - Tab. II).

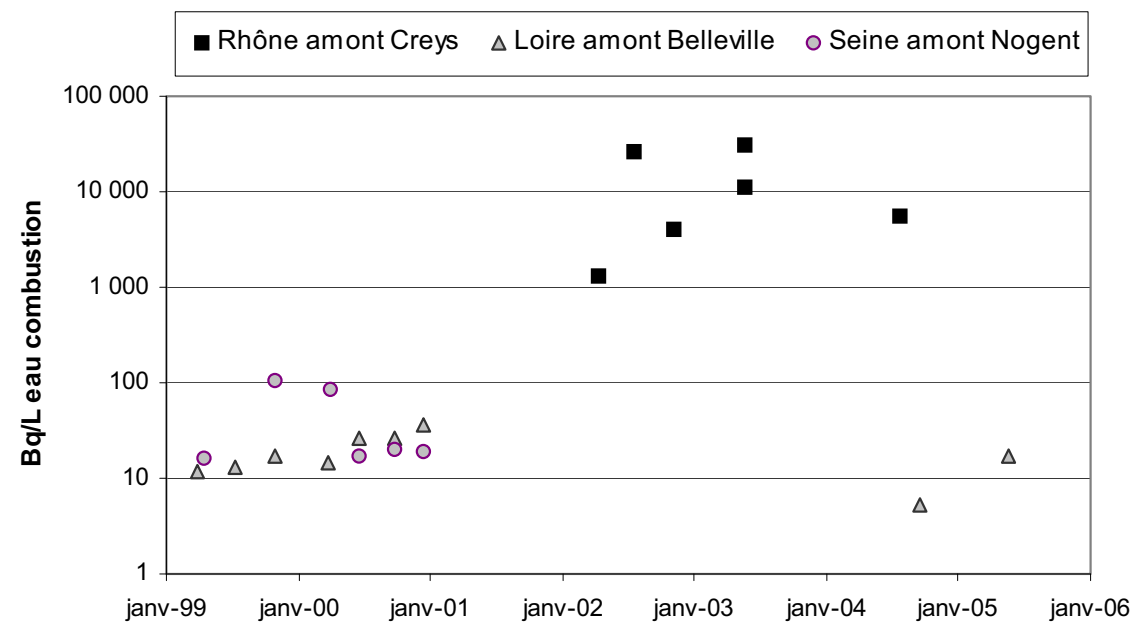

Figure 3-TOL dans les matières en suspension en amont de tous les CNPE sur le Rhône, la Seine et la Loire. OBT in suspended solids upstream of all power plants on the Rhône, Seine and Loire rivers.

Les comparaisons entre les moyennes annuelles en amont et les moyennes annuelles en aval des CNPE (Annexe - Tab. III) ne mettent pas en évidence de différences significatives dans les sédiments, ni sur l'ensemble Rhin-Rhône, ni sur 
les autres cours d'eau (Annexe - Tab. II). En revanche dans les MES, échantillonnées sur le Rhône, depuis l'amont de Creys jusqu'à l'aval de Bugey, on observe (Fig. 4) que les niveaux mesurés en amont de Creys sont supérieurs à ceux des autres stations (test de Mann-Whitney significatif, $p=0,05$ ). Cette tendance à la diminution de l'activité en allant vers l'aval confirme que l'apport de tritium provient du bassin versant amont et montre que les apports de matière organique provenant du bassin entre Creys et Bugey diluent le signal amont. Dans les matières en suspension, échantillonnées en amont et en aval de Belleville sur Loire et en amont et en aval de Nogent sur Seine de 1999 à 2001, aucune différence significative n'est mise en évidence.
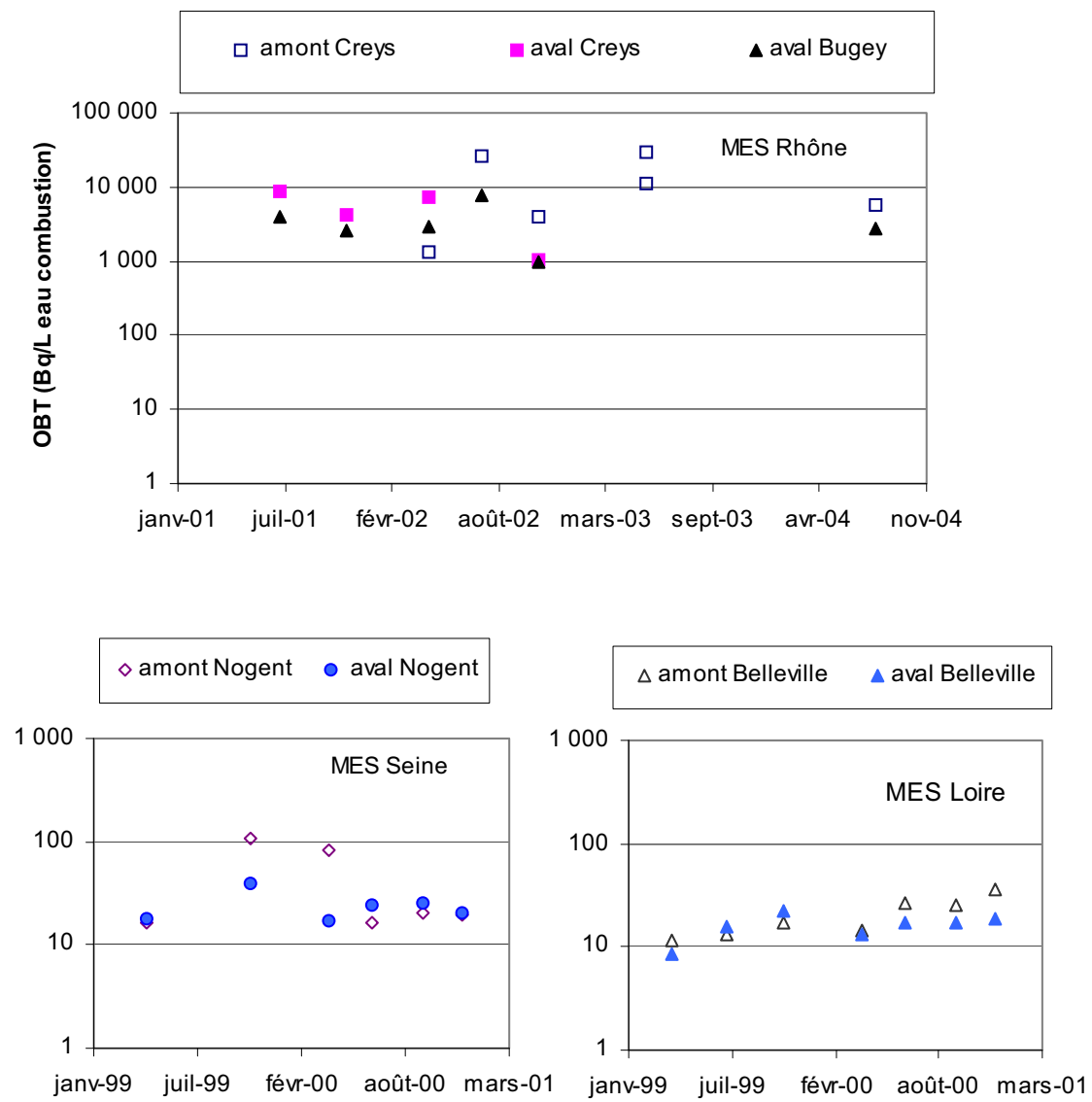

Figure 4 - Activités en TOL dans les MES décantées en amont et en aval des premiers CNPE des bassins. OBT concentrations in sedimenting particles upstream (empty marks) and downstream (full marks) of the first NPP of the basins. 


\subsection{Poissons}

195 mesures de TOL ont été réalisées dans les poissons (Fig. 5 et Annexe Tab. IV). Deux échantillons dont les activités sont inférieures à la limite de détection (environ 1 Bq. $\mathrm{L}^{-1}$ par scintillation liquide), l'un sur le Rhône en amont de Bugey en 1999, l'autre sur la Garonne en amont de Golfech en 2009 ne sont pas représentés sur la figure 5 .

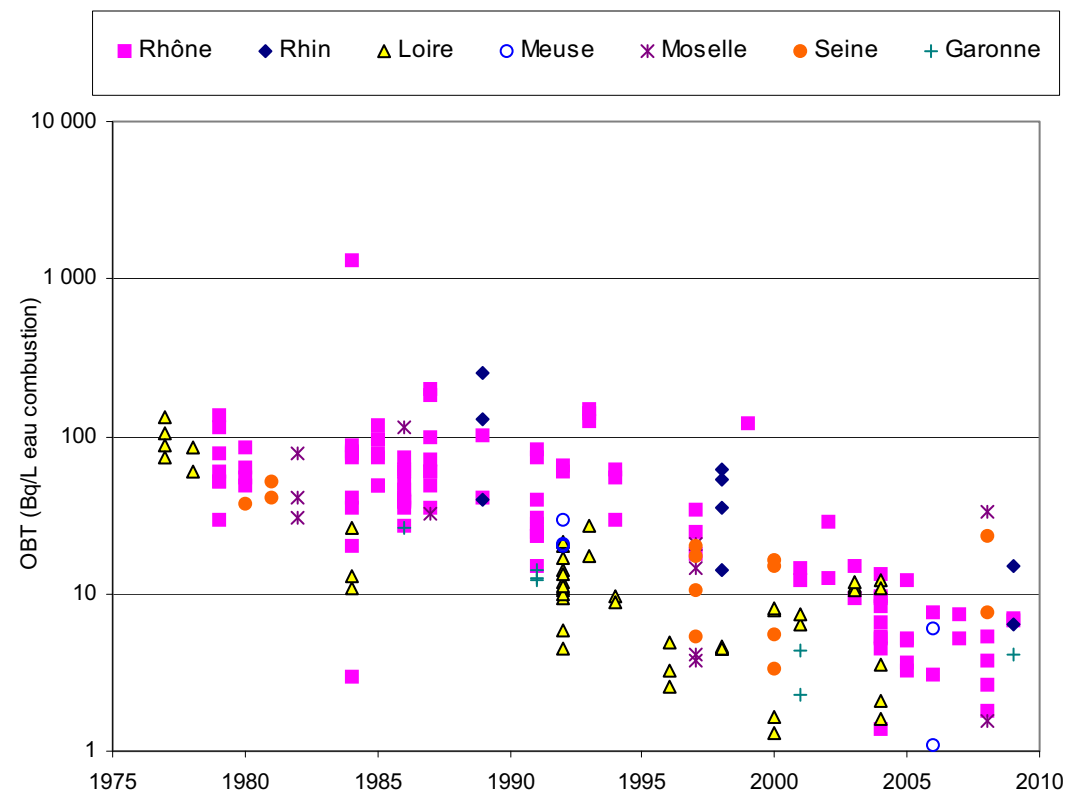

Figure 5 - Évolution des activités en TOL dans les poissons autour des CNPE.

Evolution of OBT in fish around NPP.

On constate une nette diminution des activités en TOL dans les poissons au cours des 30 dernières années, depuis des valeurs de l'ordre de 100 Bq. $\mathrm{L}^{-1}$ d'eau de combustion à la fin des années 70 vers des valeurs inférieurs à 10 Bq. $\mathrm{L}^{-1}$ d'eau de combustion actuellement. Il convient de signaler que, jusqu'en 2000, les mesures étaient faites sur le poisson entier. Il est alors possible qu'elles reflètent en partie l'activité en TOL des sédiments. En effet, la présence, dans le système digestif des poissons, de sédiments dont les activités en TOL sont globalement plus élevées, a tendance à augmenter les activités en TOL du poisson entier. On peut avoir une idée grossière de la quantité de sédiment ingéré à partir de la teneur en matière minérale du poisson (rapport entre le poids de cendres et le poids sec). 
Pour les poissons entiers analysés ici, le résidu minéral représente de 4 à $37 \%$ du poids sec. En considérant qu'un poisson éviscéré présente un résidu minéral de $10 \%$ environ, on en déduit que le sédiment peut représenter de 0 à $25 \%$ du poids du poisson. La mesure de TOL réalisée sur les muscles, à partir de 2001, permet de s'affranchir de cette source de variabilité.

Globalement sur l'ensemble des cours d'eau, les activités en TOL des poissons ont diminué selon une régression de type exponentielle, significative au seuil de 99,99 \% avec une demi-vie apparente de 6,8 ans (Annexe - Tab. I). Sur le Rhône, cette baisse est surtout sensible depuis 1999 sur la chair des poissons, (régression exponentielle de pente significative au seuil de $98 \%$, demie-vie apparente de 6 ans) attestant que les apports de tritium bio-assimilable sont globalement en diminution dans le bassin, même si les rejets d'effluents tritiés en provenance des CNPE ont augmenté durant cette période (EDF, 2008).

Sur les autres cours d'eau, cette baisse n'est significative que sur les poissons entiers, sur la période 1977-2000 (régression exponentielle de pente significative au seuil de 99,99\%, demie-vie apparente de 5,5 ans). À partir de 2000, dans les muscles de poissons cette décroissance est masquée par l'élévation des activités liée aux rejets d'effluents des CNPE.

Si l'on tient compte uniquement des échantillons collectés en amont de tout CNPE, les valeurs actuelles les plus élevées (6 à 8 Bq.L $\mathrm{L}^{-1}$ d'eau de combustion) sont observées sur le Rhin, le Rhône et la Seine, alors que sur les autres cours d'eau les activités sont de l'ordre de 1 à $2 \mathrm{~Bq} . \mathrm{L}^{-1}$ eau de combustion, proche de la limite de détection (Annexe - Tab. IV).

L'influence des rejets tritiés des CNPE est étudiée en comparant deux à deux les échantillons d'une même espèce, prélevées lors d'une même campagne à l'amont et à l'aval d'un CNPE.

Pour les cours d'eau, autres que le Rhône et le Rhin (Fig. 6), les différences amont-aval sont significatives (Annexe - Tab. II) avec un écart moyen de 7,2 Bq.L ${ }^{-1}$ entre l'aval et l'amont, alors que pour le Rhône et le Rhin (Fig. 7) les différences amont-aval ne sont pas significatives avec des valeurs amont qui dépassent en moyenne les valeurs aval (écart moyen de 44 Bq. $\mathrm{L}^{-1}$ non significatif). Plusieurs facteurs expliquent ce résultat. En premier lieu, les mesures jusqu'en 1998 étaient réalisées sur le poisson entier. Dans ces deux cours d'eau, où le sédiment est fortement marqué par le tritium organique provenant des bassins amont, on ne peut écarter une influence du contenu en sédiment du système digestif qui varie indépendamment de la position par rapport au CNPE. Cette hypothèse semble confortée par la forte hétérogénéité des teneurs en TOL des poissons entiers sur la 


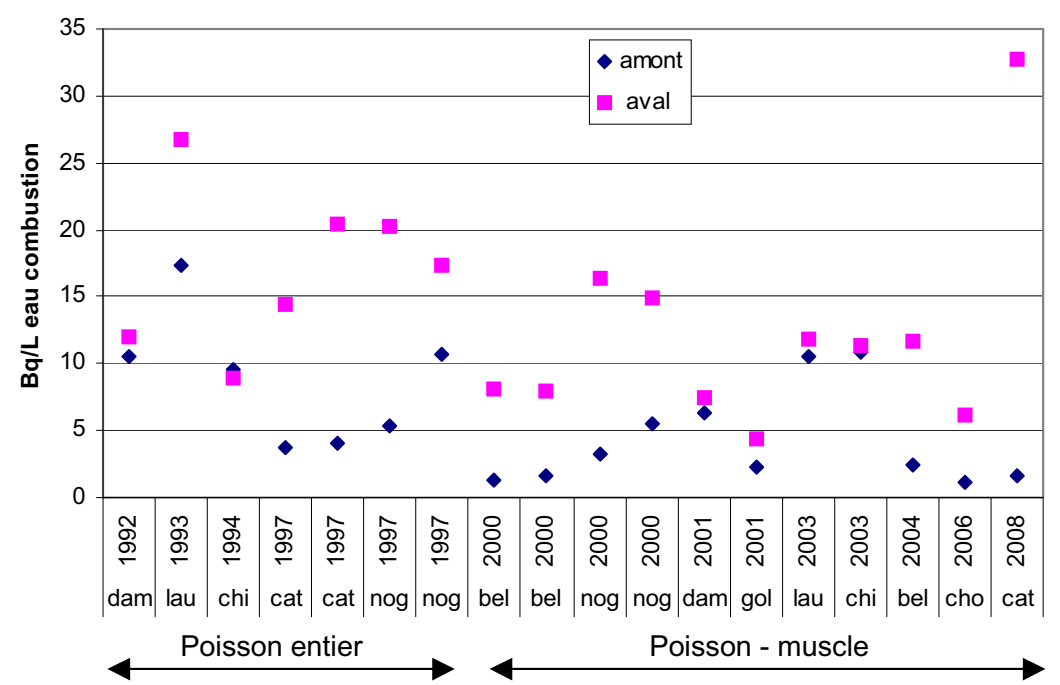

Figure 6 - Teneurs en tritium organique des poissons des cours d'eau autres que le Rhône et le Rhin. Comparaison entre l'amont et l'aval. Chaque couple de valeur correspond à la même espèce prélevée lors de la même campagne en amont et en aval d'un CNPE. Les CNPE sont représentés par les 3 premières lettres de leur nom, sauf LAU pour Saint-Laurent.

Organic tritium levels found in fish of rivers other than the Rhône and Rhine. Comparison between upstream (diamond) and downstream (square). Every couple of values corresponds to the same species collected during the same campaign, upstream and downstream of NPP. NPPs are represented by the first 3 letters of their name, except LAU which stands for SaintLaurent.

période jusqu'en 1998, avec des écarts aval- amont allant de -1300 Bq. $\mathrm{L}^{-1}$ à +90 Bq.L $\mathrm{L}^{-1}$ (Fig. 7). À partir de 1998, les mesures étant faites sur les muscles, les écarts amont-aval sont alors beaucoup plus faibles. Un deuxième facteur pouvant masquer les différences amont-aval est à attribuer à la mobilité des poissons, fonction d'une part de l'espèce concernée et d'autre part de l'absence d'obstacle (seuil, barrage) gênant leur déplacement. Sur les cours d'eau autres que le Rhône et le Rhin, le maintien d'un niveau d'eau suffisant dans les ouvrages de prise d'eau des CNPE est assuré par des seuils construits à l'aval immédiat des prises d'eau. Ces seuils qui constituent des obstacles au déplacement des poissons, entre l'amont et l'aval immédiat des sites, n'existent pas sur le Rhône et le Rhin où le maintien de la cote dans les prises d'eau est assuré par les barrages successifs aménagés pour la navigation et l'hydroélectricité, à des distances variables des CNPE.

On observe ainsi sur la figure 7 que les différences amont-aval peuvent être positives ou négatives pour l'anguille (espèce migratrice) et le hotu (qui se déplace 




Figure 7- Écart amont-aval de la teneur en tritium organique des poissons du Rhône et du Rhin. Delta TOL positif : concentration plus élevée en aval qu'en amont.

Upstream-downstream difference in organic tritium levels for Rhone and Rhine river fish. Positive values of delta OBT correspond to higher concentrations downstream than upstream. Diamond: whole fish. square: eel or nase muscle. Triangle: barbel or chub muscle.

vers l'amont pour frayer) alors que le chevesne et le barbeau présentent des teneurs en aval qui dépassent systématiquement les valeurs amont. Le test de Student (Annexe - Tab. II) conclut que les différences amont- aval sont non significatives que ce soit sur les poissons entiers, ou sur les muscles de toutes les espèces ou par groupes d'espèces. Si l'on ne tient pas compte de l'écart amont-aval à Bugey sur le barbeau en 1999 (avec des mesures singulières aussi bien en amont, où la valeur est inférieure à la limite de détection, qu'en aval où la valeur de $120 \mathrm{~Bq} . \mathrm{L}^{-1}$ eau de combustion correspond au maximum absolu sur les filets de poisson de l'ensemble des sites) la différence amont-aval sur les muscles de barbeau et de chevesne est significative ( $\mathrm{p}=0,013)$ avec un écart moyen de 3 Bq.L ${ }^{-1}$ d'eau de combustion.

Afin de mettre en évidence l'effet de l'âge des poissons sur l'activité en TOL, une campagne particulière a été réalisée en amont et en aval de Belleville/Loire sur des chevesnes de trois classes d'age (Tab. I). On note clairement l'influence des rejets sur l'activité en TOL des poissons, avec une augmentation d'environ 10 Bq. $\mathrm{L}^{-1}$ d'eau de combustion entre l'amont et l'aval. Mais, il n'y a pas d'influence de l'âge sur la teneur en TOL des chevesnes ni en aval ni en amont. Il apparaît donc que l'équilibre entre le tritium rejeté par le CNPE et le TOL des poissons est atteint dès le stade juvénile et n'est pas modifié au bout de 5 ans. 
TABLEAU I

Teneurs en TOL des muscles de chevesnes de différentes classes d’âge. OBT levels observed in the muscles of chub from different age classes.

1st column: sample collection site, 2nd column: situation: upstream or downstream of Belleville NPP, 3rd column: sample collection date, 4 th column: chub (age class), 5th column: mean length (cm), 6th column: organic tritium (Bq. $\mathrm{L}^{-1}$ combustion water), 7 th column: uncertainty (Bq.L $\mathrm{L}^{-1}$ combustion water)

\begin{tabular}{|c|c|c|c|c|c|c|}
\hline $\begin{array}{c}\text { Lieu de } \\
\text { prélèvement }\end{array}$ & $\begin{array}{l}\text { Situation/ } \\
\text { Belleville }\end{array}$ & $\begin{array}{c}\text { Date de } \\
\text { prélèvement }\end{array}$ & $\begin{array}{c}\text { Espèce } \\
\text { (classe d'âge) }\end{array}$ & $\begin{array}{c}\text { Longueur } \\
\text { moyenne } \\
(\mathrm{cm})\end{array}$ & $\begin{array}{c}\text { OBT } \\
\text { (Bq/L eau de } \\
\text { combustion) }\end{array}$ & $\begin{array}{c}\text { Incertitude } \\
\text { (Bq/L d'eau) }\end{array}$ \\
\hline Léré & amont & $21 / 09 / 04$ & $\begin{array}{l}\text { chevesne } 5^{+} \\
\text {chevesne } 1^{+} \\
\text {chevesne } \mathrm{O}^{+}\end{array}$ & $\begin{array}{l}34,7 \\
22,5 \\
17,8\end{array}$ & $\begin{array}{l}2,1 \\
3,5 \\
1,6\end{array}$ & $\begin{array}{l} \pm 0,8 \\
\pm 0,8 \\
\pm 1,8\end{array}$ \\
\hline $\begin{array}{c}2 \mathrm{~km} \text { en amont } \\
\text { du pont de } \\
\text { Beaulieu-sur- } \\
\text { Loire }\end{array}$ & aval & $22 / 09 / 04$ & $\begin{array}{l}\text { chevesne } 5^{+} \\
\text {chevesne } 1^{+}\end{array}$ & $\begin{array}{c}42 \\
26,3\end{array}$ & $\begin{array}{l}12,3 \\
10,9\end{array}$ & $\begin{array}{l} \pm 2,9 \\
\pm 1,4\end{array}$ \\
\hline
\end{tabular}

\section{Comparaison entre les activités en TOL mesurées dans les poissons et les activités en HTO calculées à partir des rejets des CNPE}

Cette comparaison est menée uniquement sur les cours d'eau où les activités en TOL dans les poissons en aval des CNPE sont statistiquement supérieures à celles en amont, c'est-à-dire sur la Loire, la Seine, la Meuse, la Moselle et la Garonne. Pour s'affranchir du niveau ambiant en TOL correspondant au marquage global du bassin versant, la grandeur considérée est la différence entre l'activité en TOL mesurée en aval et celle mesurée en amont. Cette grandeur est comparée au résultat du calcul de l'activité en HTO ajoutée par les rejets du CNPE. Les activités en HTO ajoutées dans le cours d'eau sont calculées à partir des rejets annuels en tritium de chaque CNPE (EDF, 2000, 2008) et de la médiane des débits annuels des cours d'eau récepteurs (MEDD/HYDRO, 2010). Les valeurs calculées représentent la moyenne annuelle des activités en HTO ajoutées dans le cours d'eau, après « bon mélange », c'est-à-dire en supposant que l'activité est homogène transversalement et verticalement. Il a semblé réaliste de considérer que l'activité en $\mathrm{HTO}$ « après bon mélange » était représentative de l'activité moyenne en HTO de l'eau dans laquelle se déplacent les poissons pour se nourrir.

Le tableau II présente les différences amont-aval, mesurées et calculées, pour les sites et les années où les mesures de TOL dans les poissons ont porté sur la même espèce en amont et en aval (couple de valeurs représentées sur la Fig. 6). Les valeurs présentées montrent que les augmentations d'activités en TOL dans les poissons (différences entre les mesures en amont et en aval) sont inférieures à l'augmentation d'activité en tritium du cours d'eau récepteur (calculée à partir des 
rejets), le rapport entre ces deux valeurs étant de 0,62 $\pm 0,55$. Une seule valeur de TOL dépasse de manière significative (d'un facteur 2) l'activité ajoutée dans l'eau. Il s'agit d'anguilles prélevées à Saint-Laurent en 1993. Cet écart peut être lié à une sous-estimation de l'activité dans l'eau qui est calculée après «bon mélange », alors que la pêche a lieu à proximité de l'ouvrage de rejet dans la zone où les rejets d'effluents ne sont que partiellement mélangés (à Saint-Laurent lorsque l'ouvrage de rejet était situé en berge, l'activité dans cette zone était supérieure d'un facteur 10 à l'activité calculée dans le tableau II après bon mélange. L'ouvrage de rejet a d'ailleurs été modifié en 1996 pour installer une clarinette permettant de répartir le rejet plus uniformément sur la largeur du cours d'eau).

\section{TABLEAU II}

Calcul des activités en HTO ajoutée dans le cours d'eau par les rejets des CNPE - comparaison aux écarts mesurés dans le TOL des poissons entre les stations amont et aval.

Calculation of the increases in river HTO activities resulting from power plant discharges comparison to observed differences in fish OBT between upstream and downstream stations.

\begin{tabular}{|c|c|c|c|c|c|c|}
\hline Année & Site & Espèce & $\begin{array}{c}\text { Tritium } \\
\text { dans les } \\
\text { rejets } \\
\text { liquides } \\
\text { Bq.an-1 }\end{array}$ & $\begin{array}{c}\text { Débit du } \\
\text { cours d'eau } \\
\text { m3/s }\end{array}$ & $\begin{array}{c}\text { Calcul de la } \\
\text { concentration } \\
\text { ajoutée dans } \\
\text { l'eau par CNPE }\end{array}$ & $\begin{array}{c}\text { Mesure du tritium } \\
\text { organique dans les } \\
\text { poissons (Bq. } \mathrm{L}^{-1} \text { eau } \\
\text { combustion) }\end{array}$ \\
\hline & & & & & Bq. $\mathrm{L}^{-1}$ & Delta aval-amont \\
\hline 1992 & Dampierre & anguille & $5,24 \mathrm{E}+13$ & 214,03 & 7,8 & 1,5 \\
\hline 1993 & St-Laurent & anguille & $3,30 \mathrm{E}+13$ & 241,38 & 4,3 & 9,4 \\
\hline 1994 & Chinon & anguille & $3,30 \mathrm{E}+13$ & 349,57 & 3,0 & $-0,7$ \\
\hline 1997 & Cattenom & anguille & $7,40 \mathrm{E}+13$ & 84,20 & 27,9 & 10,61 \\
\hline 1997 & Cattenom & chevaine & $7,40 \mathrm{E}+13$ & 84,20 & 27,9 & 16,38 \\
\hline 1997 & Nogent & brochet & $2,20 \mathrm{E}+13$ & 60,50 & 11,5 & 14,8 \\
\hline 1997 & Nogent & chevaine & $2,20 \mathrm{E}+13$ & 60,50 & 11,5 & 6,67 \\
\hline 2000 & Belleville & anguille & $3,90 \mathrm{E}+13$ & 203,99 & 6,1 & 6,86 \\
\hline 2000 & Belleville & barbeau & $3,90 \mathrm{E}+13$ & 203,99 & 6,1 & 6,25 \\
\hline 2000 & Nogent & anguille & $6,20 \mathrm{E}+13$ & 60,50 & 32,5 & 12,98 \\
\hline 2000 & Nogent & chevaine & $6,20 \mathrm{E}+13$ & 60,50 & 32,5 & 9,39 \\
\hline 2001 & Dampierre & barbeau & $3,50 \mathrm{E}+13$ & 214,03 & 5,2 & 1,18 \\
\hline 2001 & Golfech & gardon & $4,90 \mathrm{E}+13$ & 273,66 & 5,7 & 2,11 \\
\hline 2003 & St-Laurent & barbeau & $1,70 \mathrm{E}+13$ & 241,38 & 2,2 & 1,24 \\
\hline 2003 & Chinon & anguille & $3,50 \mathrm{E}+13$ & 349,57 & 3,2 & 0,5 \\
\hline 2004 & Belleville & chevaine & $5,60 \mathrm{E}+13$ & 203,99 & 8,7 & 9,2 \\
\hline 2006 & Chooz & chevaine & $4,90 \mathrm{E}+13$ & 91,20 & 17,0 & 5 \\
\hline 2008 & Cattenom & brême & $1,10 \mathrm{E}+14$ & 84,20 & 41,4 & 31,17 \\
\hline
\end{tabular}


Les activités en TOL des poissons peuvent être calculées en se basant sur les activités rejetées par les CNPE à partir du modèle standard d'équilibre isotopique entre compartiments de l'écosystème (IAEA, 2010). Ce modèle considère que l'activité spécifique du TOL des poissons est à l'équilibre avec l'activité spécifique moyenne en HTO de l'eau dans laquelle vit le poisson, pondéré par un facteur appelé, en anglais, « partition factor » qui tient compte de la présence d'hydrogène échangeable dans l'eau de combustion et de la discrimination isotopique qui intervient dans les premiers maillons de la chaîne trophique, lors de la photosynthèse. $\mathrm{Ce}$ « partition factor » représente donc le rapport entre l'activité en TOL des poissons, exprimée en Bq. $\mathrm{L}^{-1}$ d'eau de combustion, et l'activité en HTO dans l'eau en Bq. $\mathrm{L}^{-1}$. La valeur recommandée par l'AIEA à partir d'expérimentation de contamination de poissons en laboratoire est de 0,66 , très voisine du rapport moyen calculé à partir des valeurs des deux dernières colonnes du tableau II.

Les augmentations d'activités observées dans les poissons en aval des rejets sont donc cohérentes avec les modèles à "activité spécifique », basés sur l'équilibre isotopique entre le tritium rejeté sous forme HTO et le tritium intégré à la chair des poissons sous forme de TOL.

\subsection{Végétaux aquatiques}

Les 57 mesures de TOL dans les bryophytes et les 95 mesures sur les phanérogames sont représentées sur la figure 8. Dans les deux groupes de végétaux, on retrouve, comme pour les sédiments et les poissons, que les teneurs en TOL dans le Rhône et le Rhin sont supérieures à celles des autres cours d'eau. Ces activités reflètent en partie celles des matières en suspension retenues en surface des végétaux et difficiles à éliminer par lavage. Il est possible d'avoir une idée de la propreté des végétaux en calculant leur teneur en résidu minéral. Dans les échantillons analysés dans cet article, les teneurs en résidu minéral varient de $14 \%$ à $50 \%$, avec une moyenne de $27 \%$, pour les mousses et de $9 \%$ à $73 \%$, avec une moyenne de $25 \%$ pour les phanérogames, ce qui traduit la présence de sédiment en quantité non négligeable dans les brins. À titre indicatif, le guide sur l'utilisation des bryophytes en tant que bio-indicateur de pollution métallique (Agences de l'Eau 1998) spécifie que la teneur en résidu minéral doit être inférieure à $10 \%$ pour considérer qu'un échantillon a subi un lavage adéquat. Les mesures de TOL réalisées dans les végétaux (mousses ou phanérogames) ne représentent donc pas seulement le TOL des cellules végétales mais aussi le TOL des sédiments retenus en surface.

Si les bryophytes présentent des activités en TOL plus élevées que les phanérogames sur le Rhône, cela est dû à la répartition des lieux de collecte de ces deux types de végétaux sur le Rhône et non pas à des caractéristiques intrinsèques différentes de ces deux types de plantes vis-à-vis du tritium. En effet, de 1985 à 




Figure 8 - Évolution des activités en TOL, exprimées en Bq. $L^{-1}$ d'eau de combustion, dans les végétaux aquatiques autour des centrales nucléaires.

Evolution of organically bound tritium (OBT) activities (unit: Bq. $L^{-1}$ combustion water) in aquatic plants around nuclear power plants.

1995, les bryophytes ont été prélevées essentiellement autour des sites de Bugey et Creys dans la partie du Rhône où les sédiments présentent les activités les plus élevées en TOL, alors que sur cette même période les phanérogames proviennent de sites en aval de Lyon, de Saint-Alban à Cruas. De 1995 à nos jours, les lieux de collecte des bryophytes et des phanérogames étant distribués de façon homogène sur le cours d'eau, on ne constate plus d'écart d'activité en TOL entre les deux types de végétaux.

Globalement sur l'ensemble des cours d'eau, les activités en TOL des végétaux ont diminué selon une régression de type exponentielle, significative au seuil de 99,99\% avec une demi-vie apparente de 8 ans, en relation avec la baisse des retombées des essais nucléaires atmosphériques (Annexe - Tab. I). Sur le Rhône et le Rhin, la demie-vie apparente est de 6 ans, alors que sur les autres cours d'eau elle est de 14 ans. Cette diminution des activités en tritium plus faible sur les cours d'eau autres que le Rhône et le Rhin s'explique par des apports moindres de tritium par le bassin versant qui laissent apparaître les apports dus aux rejets des CNPE. Ce marquage des végétaux dû aux apports de tritium par les bassins amonts peut être estimé à partir des échantillons collectés en amont de tous les bassins. Sur le Rhin et le Rhône, le marquage amont est passé de 4235 Bq.L ${ }^{-1}$ d'eau de combustion en moyenne dans les années 80 à des valeurs actuelles de $33 \mathrm{~Bq} . \mathrm{L}^{-1}$ d'eau de combustion avec une forte variabilité entre échantillons (de 8 à $108 \mathrm{~Bq} . \mathrm{L}^{-1}$ d'eau de combustion). Sur les autres cours d'eau, il est passé de $28 \mathrm{~Bq} . \mathrm{L}^{-1}$ d'eau de combustion dans les années 80 à 2 Bq.L $\mathrm{L}^{-1}$ d'eau de combustion actuellement avec une faible variabilité (de 0,95 à 3,8 Bq.L ${ }^{-1}$ d'eau de combustion) (Annexe - Tab. V). 


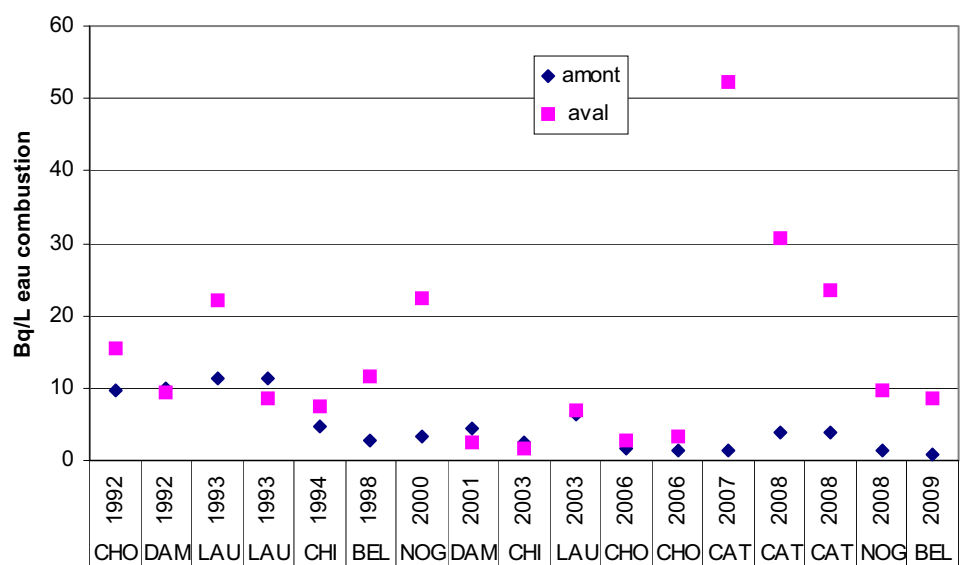

Figure 9-Teneur en TOL des végétaux aquatiques des cours d'eau autres que le Rhône et le Rhin. Comparaison amont-aval. Chaque couple de valeur correspond à la même espèce prélevée lors de la même campagne en amont et en aval d'une centrale. Les noms des centrales sont représentés par les 3 premières lettres de leur nom, sauf LAU pour Saint-Laurent.

OBT levels in aquatic plants found in rivers other than the Rhône and Rhine. Comparison between upstream (diamond) and downstream (square). Every couple of values corresponds to the same species collected during the same campaign, upstream and downstream of NPP. Power plants are represented by the first 3 letters of their name, except LAU which stands for Saint-Laurent.

Pour s'affranchir de la variabilité liée à l'activité en tritium des sédiments, les différences amont-aval sont étudiées sur les cours d'eau autres que le Rhin et le Rhône. Les comparaisons portent sur des échantillons d'une même espèce, prélevées lors d'une même campagne à l'amont et à l'aval d'un CNPE. On note globalement une augmentation des activités en TOL des végétaux en aval des CNPE (Fig. 9). Cette augmentation est statistiquement significative (Annexe Tab. II). L'écart le plus important est observé à Cattenom sur des myriophylles prélevées en 2007, à 100 m en aval de l'ouvrage de rejet, c'est-à-dire dans la zone où le rejet d'effluent n'est que partiellement mélangé dans la Moselle (dans cette zone, la dilution du rejet est inférieure d'un facteur 20 à la dilution après bon mélange). Les écarts amont-aval dans les cours d'eau à faible débit (Seine, Moselle, Meuse) sont en moyenne de 16 Bq.L $\mathrm{L}^{-1}$ d'eau de combustion alors qu'ils sont en moyenne de 2,7 Bq. $\mathrm{L}^{-1}$ d'eau de combustion en Loire. Ceci reflète d'une part les activités en tritium ajoutées dans le cours d'eau par les rejets des CNPE inversement proportionnelles aux débits des cours d'eau (Tab. II), d'autre part la nature différente des végétaux prélevés en Loire par rapport à ces autres cours d'eau. En effet, on ne trouve en Loire ni bryophytes ni phanérogames immergées, les prélèvements portent donc sur des phanérogames semi-aquatiques. Chez ces 
végétaux, l'activité en tritium est influencée par celle dans le cours d'eau et celle dans l'air, généralement proche du bruit de fond, ce qui entraîne une diminution des activités par rapport à des végétaux totalement immergés.

\section{Discussion}

L'ensemble des prélèvements et mesures réalisés de 1977 à nos jours dans l'environnement des CNPE permet d'avoir une représentation synthétique des niveaux d'activité en TOL dans les principaux bassins hydrographiques français et de préciser l'impact des rejets des CNPE.

\section{Niveau de tritium dans les zones amont des bassins hydrographiques français}

La matière organique des sédiments est la matrice qui présente les activités les plus élevées. Cet enrichissement en tritium provient pour partie de la fraction peu biodégradable de la matière organique terrestre marquée par les retombées des essais nucléaires atmosphériques. On peut considérer que ce marquage est réparti uniformément sur tout le territoire et qu'il correspond aux niveaux observés sur les cours d'eau autres que le Rhône et le Rhin en amont de tout CNPE. En utilisant l'équation exponentielle ajustée sur les mesures réalisées de 1987 à 2008, on en déduit que les niveaux en tritium de la matière organique des sédiments étaient en 1980 de l'ordre de 140 Bq.L L $^{-1}$ d'eau de combustion et ont diminué jusqu'à 5 Bq.L $\mathrm{L}^{-1}$ d'eau de combustion en 2010 (Annexe - Tab. I).



Figure 10 - Évolution des activités moyennes mensuelles en HTO dans les eaux de pluie (données AIEA/ GNIP, 2010).

Evolution of monthly-averaged HTO activities in precipitations (AIEA/GNIP, 2010 data). 
Ce marquage peut être comparé aux apports d'eau tritiée par les précipitations, voie d'entrée principale du tritium sur les bassins versants. Suite aux essais d'armes nucléaires atmosphériques, l'eau tritiée dans les précipitations en France a atteint en 1963 des activités d'environ 500 Bq.L ${ }^{-1}$ (Fig. 10) (AIEA, 2010). Les teneurs en tritium dans les précipitations ont décru ensuite de manière exponentielle d'abord avec une période de 3 ans, puis avec une période de 6 ans (Belot et al., 1996). En 1980 l'activité moyenne était de 4 à 5 Bq. $\mathrm{L}^{-1}$ dans l'eau de pluie. Elle est actuellement d'environ $0,4 \mathrm{~Bq} \cdot \mathrm{L}^{-1}$ le long de l'Océan Atlantique (proche du niveau pré-bombe) et de $1 \mathrm{~Bq} . \mathrm{L}^{-1}$ en zone continentale. Les teneurs en TOL dans la matière organique particulaire des cours d'eau en amont de tout CNPE dépassent donc d'un ordre de grandeur les teneurs dans les précipitations de la même année. En fait, en tenant compte de la décroissance radioactive, on s'aperçoit que le niveau actuel dans la matière organique des sédiments correspond au niveau observé dans les précipitations, il y a 40 ans. Ce décalage peut être considéré comme un indicateur du temps de séjour moyen du tritium dans la matière organique des sols des bassins versants. Il rejoint les estimations de Balesdent et Recous (1997), concernant les temps de résidence du carbone incorporé dans la matière organique du sol. Ces auteurs montrent que $75 \%$ de la matière organique, dans les sols cultivés français, a un temps de résidence de 40 ans.

Dans les bassins amont du Rhône et du Rhin, d'autres apports, vraisemblablement liés à l'utilisation de tritium dans la fabrication des cadrans lumineux et signaux de secours (peintures luminescentes et ampoules de tritium gazeux), s'ajoutent à ceux des retombées de tritium provenant des essais militaires. Ces industries étaient largement implantées dans le Jura français et en Suisse il y une vingtaine d'années et le demeurent encore aujourd'hui en Suisse. Gontier $e t$ al. (1992) et Pally etal. (1993) avaient déjà souligné cette zone singulière vraisemblablement soumise aux rejets de l'industrie horlogère. Jean-Baptiste $e t$ al. (2007) ont montré plus récemment que ces apports persistaient sans que l'on puisse en déterminer la composition exacte (matière organique naturelle marquée par les rejets des installations de fabrication de composés tritiés, ou rejet de composés tritiés tels que le polystyrène). À titre indicatif, les quantités de tritium achetées par l'industrie suisse de production de peinture radio-luminescente et d'ampoules de tritium gazeux sont en moyenne de $9000 \mathrm{TBq}$ par an entre 1991 et 2009 (OFSP, 2010), à comparer aux rejets des centrales de 1000 TBq par an pour l'ensemble des CNPE français (Le Guen, 2008). La majeure partie des rejets de tritium de l'industrie suisse de production de composés tritiés s'effectue par voie atmosphérique sous forme de tritium gazeux HT (Murith et al., 2006). Or cette forme chimique du tritium présente des rapports tritium/hydrogène beaucoup plus élevés que les formes HTO en raison de la concentration faible du dihydrogène $\left(\mathrm{H}_{2}\right)$ dans l'air $\left(0,5 \mathrm{ppmv}\right.$ soit $\left.10^{-5} \mathrm{~g} \mathrm{~d} \mathrm{~d}^{\prime} \mathrm{H} . \mathrm{m}^{-3}\right)$ par rapport à la vapeur d'eau atmosphérique $\left(\mathrm{H}_{2} \mathrm{O}\right)$ (en moyenne sous nos climats, $10 \mathrm{~g}$ de vapeur d'eau $/ \mathrm{m}^{3}$, soit 
$\left.1 \mathrm{~g} \mathrm{~d} \mathrm{H}^{\prime} \mathrm{m}^{-3}\right)$. Le tritium sous forme HT ne peut pas être intégré à la matière organique des végétaux par photosynthèse, mais il peut cependant être oxydé par les microorganismes du sol pour former de l'HTO et donner lieu ainsi à des activités spécifiques en tritium beaucoup plus élevés que si le tritium provenait directement de la forme HTO. De plus, certaines bactéries chémolithotrophes (Pseudomonas, Alcaligenes, par exemple) utilisent le dihydrogène comme source d'énergie pour la fixation du $\mathrm{CO}_{2}$ et peuvent ainsi incorporer le tritium sous forme HT à la matière organique. Murphy et al. (1982) dans le cadre du programme de recherche mené par le Savannah River Laboratory, identifiaient l'oxydation d'HT par les micro-organismes du sol comme la cause des niveaux élevés d'activité mesurés dans la matière organique du sol. Pour l'instant, les travaux se poursuivent pour clarifier l'origine exacte des niveaux élevés observés dans le Haut Rhône (rejets industriels de composés organiques tritiés ou produits de décomposition de la matière organique naturelle fortement marquée par les rejets industriels sous forme HTO ou HT).

Chez les poissons, comme chez les végétaux, les activités en TOL en amont de tout CNPE sont inférieures à celles des sédiments. Mais elles sont généralement supérieures aux activités en HTO des précipitations. On peut considérer que le niveau en tritium dans les précipitations donne une bonne estimation des niveaux en tritium sous forme HTO dans les eaux de surface, lorsque les eaux de surface ne sont pas alimentées principalement par des lacs ou des aquifères avec des eaux plus anciennes. Dans le cas du Rhône amont, Jean-Baptiste et al. (2007) ont montré que le tritium dans les eaux du fleuve était à l'équilibre avec le tritium dans les précipitations, malgré le séjour des eaux dans le lac Léman. Sur les autres cours d'eau, les mesures ponctuelles issues de la surveillance radioécologique des CNPE confirment cet équilibre entre eaux de surface et précipitation, sauf sur la Seine en amont du CNPE de Nogent où les activités en HTO dans le fleuve sont légèrement supérieures à celles des précipitations. La contribution de ces deux sources de tritium, que sont l'HTO atmosphérique et le TOL des sédiments, au marquage du TOL des organismes vivants aquatiques est estimée ci-après pour les poissons entiers et la chair des poissons :

- dans les cours d'eau autres que le Rhin, le Rhône et la Seine, en utilisant l'équation exponentielle ajustée sur les mesures réalisées de 1980 à 1996, sur les poissons entiers on obtient en 1980 une activité moyenne en TOL dans les poissons de $60 \mathrm{~Bq} . \mathrm{L}^{-1}$ d'eau de combustion, pour une activité moyenne en HTO des précipitations de 4 Bq. $\mathrm{L}^{-1}$ (Fig. 10) et une activité moyenne en TOL dans les sédiments de $93 \mathrm{~Bq} / \mathrm{L}$ calculée à partir de la régression exponentielle ajustée sur les sédiments de 1987 à 2008 . On peut alors en déduire que l'HTO contribue à hauteur de $37 \%$ à l'activité en TOL des poissons entiers alors que le sédiment contribue à hauteur de $63 \%$, en lien avec la présence de sédiments 
non-bioassimilables dans le système digestif. Le même calcul sur les muscles des poissons avec une moyenne en TOL de 2,7 Bq.L $\mathrm{L}^{-1}$ d'eau de combustion, sur la période 1998-2007, une moyenne dans les précipitations de 1,2 Bq.. $\mathrm{L}^{-1}$ et un niveau moyen dans la matière organique des sédiments de $13 \mathrm{~Bq} . \mathrm{L}^{-1}$ fournit une contribution de $87 \%$ due à HTO et de $13 \%$ due au TOL des sédiments qui correspond à la fraction métabolisable.

- sur le Rhône amont, on peut estimer sur la base des activités en TOL dans les muscles des poissons (moyenne de 2003 à 2009 égale à $6 \mathrm{~Bq} \cdot \mathrm{L}^{-1}$ ), de celle en HTO dans les précipitations ( $1 \mathrm{~Bq} . \mathrm{L}^{-1}$ ) et du niveau moyen en TOL des sédiments (34 $000 \mathrm{~Bq} . \mathrm{L}^{-1}$ d'eau de combustion) que la matière organique des sédiments contribue à $1 / 10000^{\mathrm{e}}$ de l'activité en TOL des poissons, en accord avec la nature très peu biodisponible du tritium organique particulaire présent dans le Haut Rhône.

- sur le Rhin et la Seine les données sont trop peu nombreuses pour quantifier précisément la contribution de chaque source.

Les niveaux mesurés par Kirchmann et Dupont (1981) dans les sédiments, les végétaux et les poissons hors influence des CNPE, en 1977-1978, sont cohérents avec ceux présentés ici pour des bassins où les organismes vivants sont exposés à deux formes distinctes de tritium : l'eau tritiée avec une activité relativement faible qui correspond à celle des précipitations de l'année de prélèvement, et la matière organique des sédiments qui provient de la matière organique des sols dont une partie s'est formée lorsque les retombées atmosphériques des essais nucléaires atmosphériques étaient maximales.

Les activités dans la matière organique des poissons supérieures aux activités en eau tritiée des eaux de surface ne traduisent pas l'existence d'un phénomène de bioaccumulation ${ }^{4}$ à partir d'HTO ; elles correspondent à un état d'équilibre entre deux sources de tritium d'activité spécifique différente : l'HTO qui entre dans la chaîne alimentaire des poissons par l'intermédiaire du phytoplancton et du zooplancton, le TOL des sédiments dont une partie peut être métabolisée par les bactéries et le zoobenthos, et servir de nourriture aux poissons.

\section{Impact des rejets en tritium des $\mathrm{CNPE}$}

Les comparaisons entre les activités en TOL mesurées en amont et en aval des CNPE sur les poissons ou les végétaux font apparaître des écarts plus importants dans les cours d'eau à faible débit où les activités moyennes annuelles en HTO

\footnotetext{
4 La définition du terme «bioaccumulation » est celle retenue par le groupe de réflexion sur le tritium organisé par l'ASN (Smeesters et Masse 2010), c'est-à-dire: la bioaccumulation résulte d'un phénomène d'accumulation progressive d'un contaminant ou d'une substance toxique dans un organisme, à partir de diverses sources, y compris l'atmosphère, l'eau et les aliments, jusqu'à l'obtention d'un état d'équilibre entre accumulation et élimination de la substance, avec une concentration dans l'organisme supérieure à celle des sources de contamination.
} 
sont plus élevées que dans les grands fleuves, ainsi que dans les zones proches du point de rejet lorsque l'effluent n'est que partiellement mélangé. Que ce soit dans les poissons, les végétaux ou les sédiments, aucune augmentation des activités en TOL supérieure aux activités ajoutées dans l'eau par les rejets des CNPE n'est constatée. On peut donc en déduire qu'aucun phénomène de bioaccumulation ${ }^{4} \mathrm{du}$ tritium provenant des rejets des CNPE n'est mis en évidence. Ces résultats confirment les conclusions des expérimentations menées en laboratoire par Kirchmann et al. (1979) sur des effluents de CNPE et des effluents de laboratoire de synthèse de molécules marquées. Ces auteurs ont montré que le tritium des effluents de laboratoire de synthèse de molécules marquées était préférentiellement incorporé à la matière organique des algues avec des rapports entre le tritium dans l'eau de combustion et le tritium dans l'eau du milieu largement supérieur à 10 alors que dans le cas des effluents des centrales REP de Chooz (France) et de Tihange (Belgique), ce rapport était inférieur à 1 . Ils concluent que la fraction soluble des effluents rejetés par les centrales REP est constituée uniquement d'eau tritiée. Ce n'est pas le cas des effluents rejetés par les laboratoires de synthèse de molécules marquées, qui contiennent vraisemblablement des molécules hydrogénées fortement enrichies en tritium. Ce sont ces molécules qui sont responsables de l'augmentation des activités dans la matière organique des algues et non pas un phénomène de bioaccumulation à partir de l'eau tritiée. L'enrichissement en tritium de ces molécules n'ayant pas été estimé, il n'est pas possible de calculer le rapport entre le TOL des algues et le TOL des molécules marquées. Seul ce rapport pourrait permettre de conclure à l'existence éventuelle d'une bioaccumulation. À cet égard, il convient de noter que la baie de Cardiff où des activités élevées ont été observées dans les poissons, les mollusques et les crustacés, est exposée aux rejets d'une usine de fabrication de molécules biologiques marquées au tritium. Là aussi, en l'absence de données sur le taux d'enrichissement en tritium des molécules marquées présentes dans les rejets, il n'est pas possible de calculer le rapport entre l'activité en TOL dans les organismes marins et l'activité en TOL des molécules marquées. Ces résultats montrent cependant le rôle de bio-indicateurs que peuvent jouer les organismes vivants pour mettre en évidence la présence éventuelle de formes organiques du tritium dans les rejets.

Même si les rejets de tritium des CNPE peuvent contribuer aux niveaux observés dans les MES et les sédiments par incorporation du tritium rejeté sous forme HTO au phytoplancton et au phytobenthos, puis transfert au zoobenthos, aucune différence significative n'est mise en évidence entre l'amont et l'aval des CNPE, probablement parce que l'influence des rejets est masquée par les apports du bassin versant amont. L'étude des MES et des sédiments de l'estuaire de la Loire (Siclet, 2001) montrait également qu'à l'échelle de l'ensemble du bassin de la Loire, soumis aux rejets de 5 CNPE, les niveaux en TOL des MES et des sédiments n'étaient pas influencés par les rejets des CNPE alors qu'à l'inverse les 
activités en HTO dans l'estuaire coïncidaient parfaitement avec les calculs basés sur les rejets des CNPE.

\section{Conclusion}

Malgré l'hétérogénéité des activités dans le milieu naturel et l'évolution des méthodes de prélèvement et d'analyses, il a été possible grâce aux séries chronologiques longues constituées à partir des données sur les activités en TOL dans l'environnement des CNPE, d'exploiter statistiquement ces résultats et de dégager les enseignements suivants sur le cycle du tritium organique dans l'environnement.

Au cours des trente dernières années, les activités en TOL ont diminué de façon exponentielle dans tous les compartiments des écosystèmes aquatiques, en relation avec la décroissance radioactive du tritium produit lors des essais nucléaires atmosphériques des années 60. Les niveaux atteints aujourd'hui dans les zones non influencées par des rejets industriels se rapprochent des activités antérieures aux essais nucléaires. Dans ces zones, les activités en TOL dans les sédiments sont supérieures aux activités mesurées dans les poissons et les végétaux, elles-mêmes supérieures à celles des eaux de surface. Les niveaux observés résultent de l'exposition des organismes aquatiques à deux sources distinctes de tritium: l'HTO des précipitations, le TOL des sols. La matière organique des sols constitue un réservoir de tritium provenant de la décomposition des végétaux terrestres où le temps de résidence du tritium peut être estimé à 40 ans. Il alimente le compartiment sédimentaire des cours d'eau et évolue indépendamment de l'eau tritiée. Ce tritium organique des sédiments est ensuite piégé en surface des végétaux et dans les viscères des poissons ; une fraction est métabolisée par les bactéries et le zoobenthos qui l'intègrent ainsi à la matière organique vivante. La contribution de la matière organique des sédiments au niveau du TOL observé dans la chair des poissons varie en fonction de la nature des composés organiques présents dans le sédiment. Elle est de l'ordre de $10 \%$ s'il s'agit de matière organique issue de substances humiques naturelles marquées par les retombées atmosphériques de tritium, elle est plus faible d'un facteur 1000 si le TOL provient de composés organiques tritiés rejetés par l'industrie horlogère (cas des bassins du Rhône et du Rhin).

L'influence des rejets de tritium des CNPE est clairement perceptible dans le TOL des végétaux aquatiques et des poissons des cours d'eau autres que le Rhône et le Rhin : les écarts amont-aval sont en moyenne de 10 Bq.L . $^{-1}$ d'eau de combustion, avec des variations en fonction de la distance entre le rejet et la zone de prélèvement (dilution partielle de l'effluent à proximité du rejet), et en fonction du débit du cours d'eau (concentration en tritium inversement proportionnelle au débit moyen du cours d'eau). Que ce soit dans les poissons, les végétaux ou les sédiments, aucune augmentation des activités en TOL supérieure aux activités ajoutées dans l'eau par les rejets des CNPE n'est constatée, témoignant de l'absence 
de composés organiques bioaccumulables dans les rejets des CNPE et du caractère enveloppe de l'hypothèse d'équilibre isotopique entre le TOL des poissons et l'HTO.

Quelle que soit la source de contamination en tritium de l'environnement (production naturelle, retombées des essais nucléaires atmosphériques, rejets de l'industrie de l'horlogerie et des peintures luminescentes, rejets des centrales nucléaires), les résultats obtenus sur les cours d'eau français montrent que les activités en TOL dans les organismes vivants exprimés en Bq. $\mathrm{L}^{-1} \mathrm{~d}$ 'eau de combustion ne sont jamais supérieures à la source de contamination, exprimées en $\mathrm{Bq} . \mathrm{L}^{-1}$ d'eau pour HTO ou en Bq.L $\mathrm{L}^{-1}$ d'eau de combustion pour les composés organiques. Les seuls cas d'apparents déséquilibres sont dus à l'existence d'une autre source de tritium d'activité spécifique plus élevée. Ceci démontre l'intérêt d'un réseau d'observation pérenne de l'activité en TOL des organismes vivants en tant qu'indicateurs de la présence éventuelle de formes chimiques du tritium autre que l'eau tritiée.

À l'avenir pour que ces analyses continuent à être exploitables en tant qu'outil de suivi des activités en TOL, il convient, notamment pour les faibles niveaux dans l'environnement, d'améliorer la métrologie du TOL et d'harmoniser la quantification du TOL non échangeable depuis le choix des espèces et des fractions échantillonnés, leurs modes de conservation et de traitement, jusqu'au comptage et l'expression des résultats. En parallèle, il nous semble important d'inclure, dans la réflexion sur l'optimisation de la surveillance du TOL dans l'environnement, des objectifs de limitation des pressions de prélèvement sur les écosystèmes.

ANNEXE - TABLEAU I

Ajustement des tendances d'évolution temporelle du TOL - Les régressions ont été soumises à un test de signification de la pente : lorsque la pente est significativement différente de zéro $(p<0,05)$, elle est indiquée en gras.

Fit of OBT temporal trends. Regressions were tested for slope significance: when the slope is significantly different from zero $(p<0.05)$, the slope is in bold.

\begin{tabular}{|c|c|c|c|}
\hline Matrice & Cours d'eau & Pente $\left(a^{-1}\right)$ & $\begin{array}{c}\text { Probabilité } \\
\text { P }\end{array}$ \\
\hline sédiments & Rhin-Rhône & $-0,039$ & 0,008 \\
\hline sédiments & hors Rhin-Rhône & $-0,096$ & 5 E-9 \\
\hline sédiments & $\begin{array}{l}\text { hors Rhin-Rhône } \\
\text { amont tout CNPE }\end{array}$ & $-0,113$ & 0,026 \\
\hline poissons & tous & $-0,101$ & $4 \mathrm{E}-30$ \\
\hline poissons (filets) & tous & $-0,069$ & 0,02 \\
\hline poissons entiers & Rhône & $-0,030$ & 0,08 \\
\hline poissons (filets) & Rhône & $-0,114$ & 0,038 \\
\hline poissons entiers & hors Rhin-Rhône & $-0,125$ & $5 \mathrm{E}-13$ \\
\hline poissons (filets) & hors Rhin-Rhône & $-0,044$ & 0,84 \\
\hline poissons & $\begin{array}{l}\text { hors Rhin-Rhône } \\
\text { amont tout CNPE }\end{array}$ & $-0,131$ & $1,7 \mathrm{E}-11$ \\
\hline végétaux aquatiques & tous & $-0,084$ & $4 \mathrm{E}-7$ \\
\hline végétaux aquatiques & Rhin-Rhône & $-0,106$ & $2 \mathrm{E}-8$ \\
\hline végétaux aquatiques & hors Rhin-Rhône & $-0,051$ & $8 \mathrm{E}-4$ \\
\hline
\end{tabular}




\section{ANNEXE - TABLEAU II}

Tests statistiques appliqués aux comparaisons de séries de données de TOL - Le test de Student apparié a été utilisé lorsque les jeux de données le permettait, sinon les comparaisons ont été menées avec le test non paramétrique de Mann-Whitney. Lorsque la différence est significative $(p<0,05)$, la valeur de p est en gras Statistical tests applied to the comparison of TOL data sets. A paired Student test was used when the data set allowed it, otherwise comparisons were performed with the Mann-Whitney non parametric test. In case of a significant difference between data sets $(p<0.05)$, the $p$ value is in bold.

\begin{tabular}{|c|c|c|c|c|c|}
\hline \multirow[b]{2}{*}{ Matrice } & \multirow[b]{2}{*}{ Cours d'eau } & \multirow[b]{2}{*}{ TOL - série 1} & \multirow[b]{2}{*}{ TOL - série 2} & \multicolumn{2}{|c|}{ Valeur de $p$} \\
\hline & & & & $\begin{array}{l}\text { Test de } \\
\text { Student } \\
\text { apparié }\end{array}$ & $\begin{array}{c}\text { Test de Mann } \\
\text { de Whitney }\end{array}$ \\
\hline sédiments & Rhin-Rhône & $\begin{array}{l}\text { amont - } 25 \text { moyennes } \\
\text { annuelles (tableau III) }\end{array}$ & $\begin{array}{c}\text { aval - } 25 \text { moyennes } \\
\text { annuelles (tableau III) }\end{array}$ & 0,2 & \\
\hline sédiments & hors Rhin-Rhône & $\begin{array}{l}\text { amont - } 16 \text { moyennes } \\
\text { annuelles (tableau III) }\end{array}$ & $\begin{array}{l}\text { aval - } 16 \text { moyennes } \\
\text { annuelles (tableau III) }\end{array}$ & 0,3 & \\
\hline MES & Rhône & $\begin{array}{l}\text { amont Creys - } 6 \\
\text { mesures (Figure 4) }\end{array}$ & $\begin{array}{c}\text { aval Creys - } 4 \text { mesures } \\
\text { aval Bugey - } 6 \text { mesures } \\
\text { (Figure } 4 \text { ) }\end{array}$ & & 0,05 \\
\hline MES & Loire & $\begin{array}{l}\text { amont belleville - } 7 \\
\text { mesures (Figure 4) }\end{array}$ & $\begin{array}{l}\text { aval Belleville - } 7 \\
\text { mesures (Figure } 4 \text { ) }\end{array}$ & 0,92 & \\
\hline MES & Seine & $\begin{array}{l}\text { amont Nogent - } 6 \\
\text { mesures (Figure } 4 \text { ) }\end{array}$ & $\begin{array}{l}\text { aval Nogent - } 6 \\
\text { mesures (Figure 4) }\end{array}$ & 0,88 & \\
\hline MES & Loire-Seine & $\begin{array}{l}\text { Loire - amont } \\
\text { Belleville - } 7 \text { mesures } \\
\text { (Figure 4) }\end{array}$ & $\begin{array}{l}\text { Seine - amont Nogent - } \\
6 \text { mesures (Figure 4) }\end{array}$ & & 0,32 \\
\hline poissons & Rhin-Rhône & $\begin{array}{l}\text { amont }-25 \text { mesures } \\
\text { (Figure } 7 \text { ) }\end{array}$ & $\begin{array}{l}\text { aval }-25 \text { mesures } \\
\text { (Figure 7) }\end{array}$ & 0,41 & \\
\hline poissons & hors Rhin-Rhône & $\begin{array}{l}\text { amont - } 18 \text { mesures } \\
\text { (Figure 6) }\end{array}$ & $\begin{array}{l}\text { aval - } 18 \text { mesures } \\
\text { (Figure 6) }\end{array}$ & 0,00037 & \\
\hline poissons entiers & Rhin-Rhône & $\begin{array}{l}\text { amont - } 11 \text { mesures } \\
\text { (Figure } 7)\end{array}$ & $\begin{array}{l}\text { aval - } 11 \text { mesures } \\
\text { (Figure } 7 \text { ) }\end{array}$ & 0,37 & \\
\hline poissons muscles & Rhin-Rhône & $\begin{array}{l}\text { amont }-14 \text { mesures } \\
\text { (Figure } 7 \text { ) }\end{array}$ & $\begin{array}{l}\text { aval - } 14 \text { mesures } \\
\text { (Figure 7) }\end{array}$ & 0,35 & \\
\hline $\begin{array}{l}\text { poissons muscles } \\
\text { (sans barbeau } \\
\text { Bugey 1999) }\end{array}$ & Rhin-Rhône & $\begin{array}{l}\text { amont - } 13 \text { mesures } \\
\text { (Figure } 7)\end{array}$ & $\begin{array}{l}\text { aval - } 13 \text { mesures } \\
\quad \text { (Figure } 7 \text { ) }\end{array}$ & 0,91 & \\
\hline $\begin{array}{l}\text { anguille- holu } \\
\text { muscles }\end{array}$ & Rhin-Rhône & $\begin{array}{l}\text { amont }-5 \text { mesures } \\
\text { (Figure } 7 \text { ) }\end{array}$ & $\begin{array}{l}\text { aval }-5 \text { mesures } \\
\quad \text { (Figure } 7 \text { ) }\end{array}$ & 0,09 & \\
\hline $\begin{array}{c}\text { barbeau-chevesne } \\
\text { muscles }\end{array}$ & Rhin-Rhône & $\begin{array}{l}\text { amont }-9 \text { mesures } \\
\text { (Figure } 7 \text { ) }\end{array}$ & $\begin{array}{l}\text { aval }-9 \text { mesures } \\
\quad \text { (Figure } 7 \text { ) }\end{array}$ & 0,25 & \\
\hline $\begin{array}{l}\text { barbeau-chevesne } \\
\text { muscles (sans } \\
\text { Bugey 1999) }\end{array}$ & Rhin-Rhône & $\begin{array}{l}\text { amont - } 8 \text { mesures } \\
\text { (Figure } 7)\end{array}$ & $\begin{array}{l}\text { aval - } 8 \text { mesures } \\
\quad \text { (Figure } 7 \text { ) }\end{array}$ & 0,013 & \\
\hline $\begin{array}{l}\text { végétaux } \\
\text { aquatiques }\end{array}$ & hors Rhin-Rhône & $\begin{array}{l}\text { amont - } 17 \text { mesures } \\
\text { (Figure 9) }\end{array}$ & $\begin{array}{l}\text { aval - } 17 \text { mesures } \\
\text { (Figure 9) }\end{array}$ & 0,012 & \\
\hline
\end{tabular}




\section{ANNEXE - TABLEAU III}

Activités en TOL dans les sédiments en amont et en aval des CNPE - Pour chaque site, $1^{\text {re }}$ ligne : année de prélèvement, $2^{\mathrm{e}}$ ligne : position par rapport à la centrale, $3^{\mathrm{e}}$ ligne : moyennes annuelles de l'activité en TOL en Bq. $\mathrm{L}^{-1}$ d'eau de combustion, $4^{\mathrm{e}}$ ligne : nombre d'échantillons ayant servi au calcul de la moyenne, texte hors cadre : traitements particuliers subis par les échantillons (sauf indication contraire, les mesures ont été faites par scintillation liquide jusqu'en 2000 et par spectrométrie de masse sur ${ }^{3} \mathrm{He}$ à partir de 2001). En italique : MES. En grisé : périodes antérieures au démarrage de la centrale.

OBT concentrations in sediments upstream and downstream of power plants. 1st line: year of collection, 2nd line: position relative to the power plant, $3 \mathrm{rd}$ line: yearly mean of $\mathrm{OBT}$ activity in $\mathrm{Bq} \cdot \mathrm{L}^{-1}$ combustion water, 4th line: number of samples included in the calculation of the mean, text outside box: special treatment of the samples (unless otherwise mentioned, all the measures were made by liquid scintillation until 2000 and by mass-spectrometry of ${ }^{3} \mathrm{He}$ from 2001 onward). Italics: suspended solids. Grey background: series corresponding to periods before the powerplant start-up.

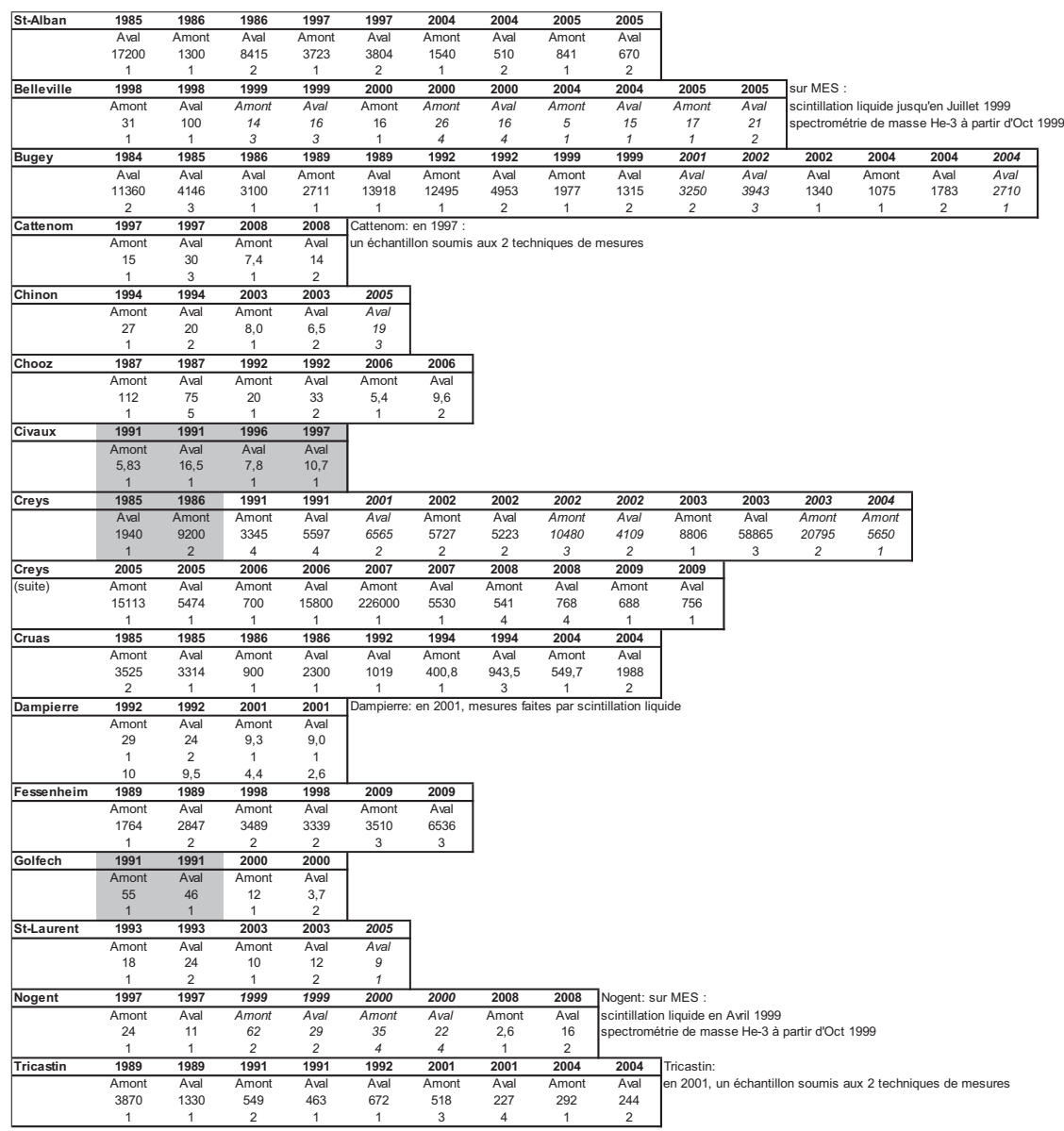




\section{ANNEXE - TABLEAU IV}

Activités en TOL chez les poissons en amont et en aval des CNPE - Pour chaque site, $1^{\text {re }}$ ligne : année de prélèvement, $2^{\mathrm{e}}$ ligne : position par rapport à la centrale, $3^{\mathrm{e}}$ ligne : moyennes annuelles de l'activité en TOL prélèvement, $2^{\mathrm{C}}$ ligne : position par rapport a la centrale, $3^{\mathrm{C}}$ ligne : moyennes annuelles de l'activité en TOL fractionnements particuliers subis par les échantillons (sauf indication contraire, toutes les mesures à partir de 2000, ont été faites sur les muscles des poissons, alors qu'elles étaient réalisées sur les poissons entiers de 2000, ont été faites sur les muscles des poissons, alors qu'elles étaient réalisées sur les poissons entiers
auparavant). En grisé : séries correspondant aux périodes antérieures au démarrage de la centrale. Toutes les auparavant). En grisé : séries correspondant aux périodes antérieures au démarrage de la centrale.
mesures rapportées ici ont été faites par scintillation liquide sur l'eau de combustion.

mesures rapportées ici ont été faites par scintillation liquide sur l'eau de combustion.
OBT concentrations in fish upstream (amont) and downstream (aval) of power plants. For every site 1st line year of collection, 2 nd line: position relative to the power plant 3rd line: yearly mean of OBT activity in Bq. $\mathrm{L}^{-1}$ combustion water, 4th line: number of samples included in the calculation of the mean, 5 th line: special fractionations of the samples (unless otherwise mentioned, all the measures from 2000 onward were done on fish muscles, whereas they were previously done on whole fish). Grey background: series corresponding to periods before the powerplant start-up. All the measurements reported here were made by liquid scintillation.

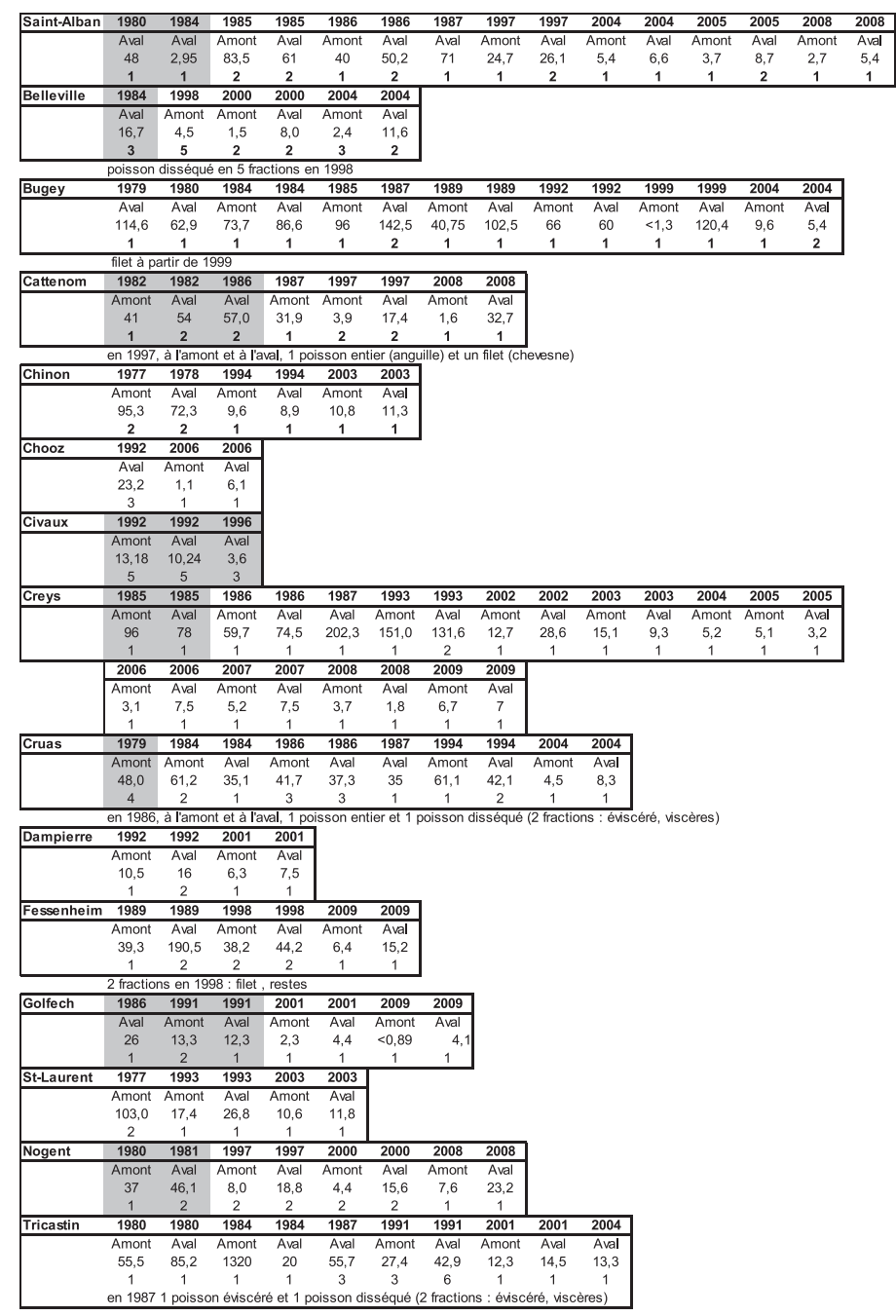




\section{ANNEXE - TABLEAU V}

Activités en TOL dans les végétaux aquatiques en amont et en aval des CNPE - Pour chaque site, $1^{\text {re }}$ ligne : année de prélèvement, $2^{\mathrm{e}}$ lione : position par rapport à la centrale, $3^{\mathrm{e}}$ ligne : moyennes annuelles de l'activité en TOL en Bq.L ${ }^{-1}$ d'eau de combustion, $4^{\mathrm{e}}$ ligne : nombre d'échantillons ayan servi au calcul de la moyenne. En caractère normal : les bryophytes. En italique : les phanérogames. servi au calcul de la moyenne. En caractère normal : les bryophytes. En italique : les phanérogames. faites par scintillation liquide, sauf à Creys en 2002, et 2005 sur les bryophytes et en 2003 sur les phanérogames où les analyses sont faites par spectrométrie de masse de ${ }^{3} \mathrm{He}$

OBT concentrations in aquatic plants upstream (amont) and downstream (aval) of power plants. For every site, 1st line: year of collection, 2 nd line: position relative to the power plant 3rd line: yearly mean of OBT activity in Bq.L $\mathrm{L}^{-1}$ combustion water, 4 th line: number of samples included in the calculation of the mean. Regular font: bryophytes, italics : phanerogams. Grey background: series corresponding to the mean. Regular font: bryophytes, italics : phanerogams. Grey background: series corresponding to
periods before the powerplant start-up. All the measurements reported here were made by liquid scintillation, except in Creys on bryophytes in 2002 and 2005, and on phanerogams in 2003 where analysis were done by mass spectrometry on ${ }^{3} \mathrm{He}$.

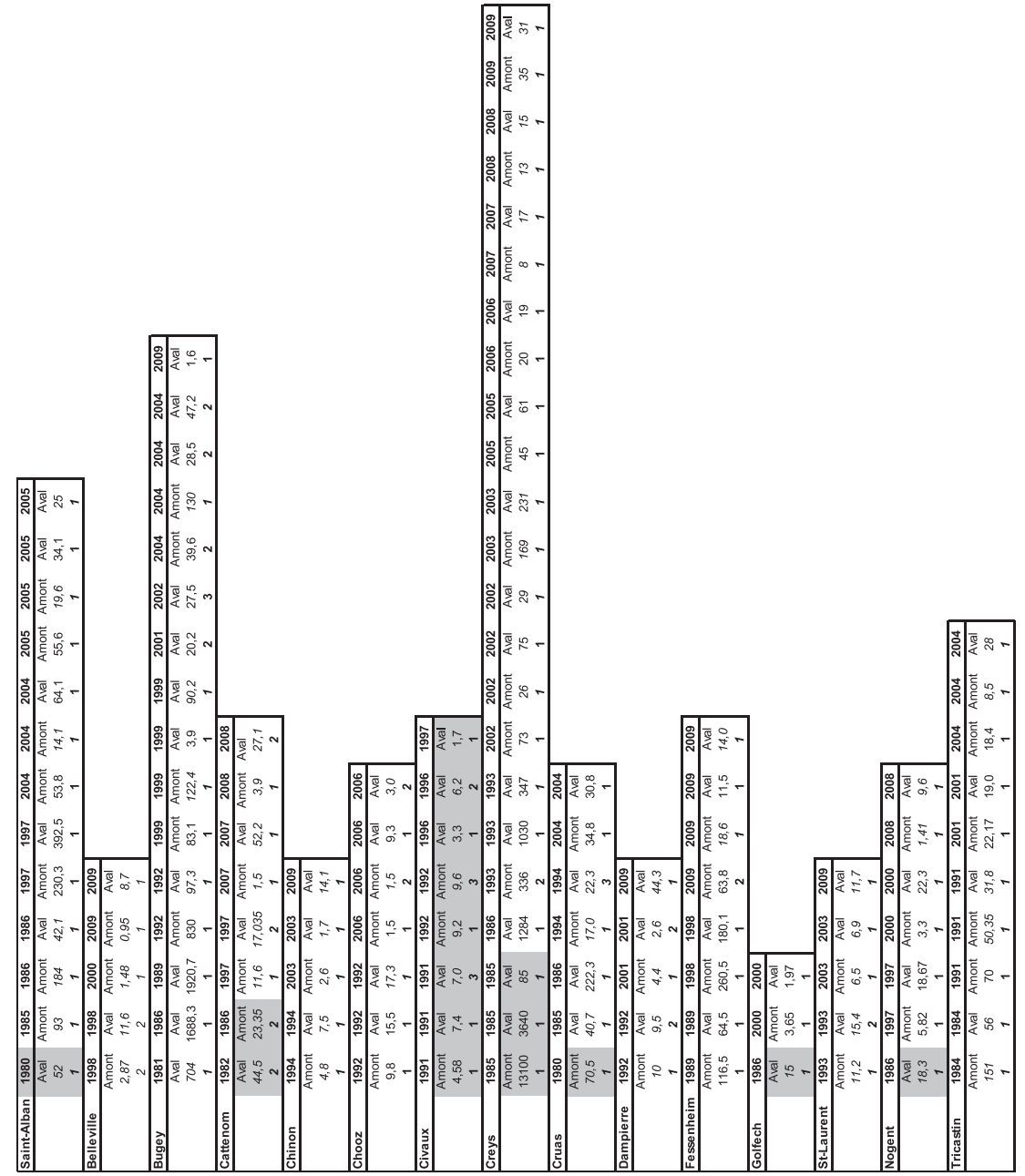




\section{RÉFÉRENCES}

Abril G., Nogueira M., Etcheber H., Cabeçadas G., Lemaire E., Brogueira M.J. (2002) Behaviour of Organic Carbon in Nine Contrasting European Estuaries, Estuarine, Coastal and Shelf Science 54, 241-262.

Agences de l'Eau (1998) Les bryophytes aquatiques comme outil de surveillance de la contamination des eaux courantes par les micropolluants métalliques : concept, méthodologie et interprétation des données, Étude Inter-Agences $n^{\circ} 55$

AIEA/GNIP (2010) Global Network of Isotopes in Precipitations - http://isohis.iaea.org/.

Antonelli C. (2007) Bilan radioécologique décennal de l'environnement proche du Centre Nucléaire de Production d'Électricité de Saint-Laurent-des-Eaux (2003), Rapport IRSN/SESURE n 2007 $51,116 \mathrm{p}$.

Antonelli C. (2008) Bilan radioécologique décennal de l'environnement proche du centre nucléaire de production d'électricité de Chinon-Avoine (2003), Rapport IRSN/SESURE n 2008-07, 122 p.

Antonelli C. (2009) Bilan radioécologique décennal de l'environnement proche du Centre Nucléaire de Production d'Électricité de Cruas (2004), Rapport IRSN/DEI/SESURE n 2009-07, 145 p.

Antonelli C. (2010) Bilan radioécologique décennal de l'environnement proche du Centre Nucléaire de Production d'Électricité de Cattenom (2008), Rapport IRSN/DEI/SESURE n 2010-07, 149 p.

Baglan N., Ansoborlo E., Cossonnet C., Fouhal L., Deniau I., Mokili M., Fourré E., Olivier A. (2010) Métrologie du tritium dans différentes matrices : cas du tritium organiquement lié (TOL), Radioprotection 45, 369-390.

Balesdent J., Recous S. (1997) Les temps de résidence du carbone et le potentiel de stockage de carbone dans quelques sols cultivés français, Can. J. Soil Sci. 77, 187-193.

Beaugelin K., Thirion J.P. (1997) Bilan radioécologique décennal du Centre Nucléaire de Production d'Électricité de Creys-Malville (1996), Document IPSN/DPRE/SERE 97-029, 199 p.

Belot Y., Roy M., Métivier H. (1996) Le tritium de l'environnement à l'homme. EDP Sciences, Les Ulis (France).

Blaylock B.G., Hoffman F.O., Frank M.L. (1986) Tritium in the aquatic environment, Radiat. Prot. Dosim. 16, 65-71.

Boyer C., Vichot L., Fromm M., Losset Y., Tatin-Froux F., Guétat P., Badot P.M. (2009) Tritium in plants: A review of current knowledge, Environmental and Experimental Botany 67, 34-51.

Calmon P. (2011) Bilan radioécologique décennal de l'environnement proche du Centre Nucléaire de Production d'Électricité de Fessenheim (2009), Rapport IRSN/DEI/SESURE sous presse, $150 \mathrm{p}$.

Camus H., Carrere D., Simeon C. (1987) Utilisation des mesures de tritium pour la surveillance de l'environnement, Rapport CEA-N-2517, 190 p.

Cossonnet C., Neiva Marques A.M., Gurriaran R. (2009) Experience acquired on environmental sample combustion for organically bound tritium measurement, Appl. Radiat. Isotopes 67, 809811.

Descamps B., Vray F., Haristoy D., Beneito A. (2000) Point zéro actualisé du site de Civaux, Document IRSN/DPRE/SERNAT 2000-08, 128 p. et annexes.

Diabate S., Strack S. (1993) Organically bound tritium, Health Phys. 65, 698-712.

Duffa C. (2003) Bilan radioécologique décennal du Centre Nucléaire de Production d'Electricité de Dampierre (2001), Rapport DPRE/SERNAT 2003-10, 111 p.

EDF (2000) Nucléaire et Environnement 2000 EDF Division Production Nucléaire.

EDF (2008) Nucléaire et Environnement 2008 EDF Division Production Nucléaire. 
Fontugne M., Sadouni N., Saliot A. (2002) ${ }^{14} \mathrm{C}$ activity in dissolved mineral carbon and identified organic matter in the Loire estuary (France), Ecorad 2001, Aix-en-Provence, Sept. 3-7, 2001, Radioprotection - Colloques 37, C1, 775-780.

Foulquier L., Pally M. (1982) Données sur la teneur en tritium lié de poissons de grands fleuves français, Annales de l'Association Belge de Radioprotection 7, 259-281.

Gontier G. (2004) Bilan radioécologique décennal du centre Nucléaire de Production d'Électricité de Golfech (2000), Rapport IRSN/DEI/SESURE n ${ }^{\circ} 03-12,120 \mathrm{p}$.

Gontier G. (2006) Deuxième bilan radioécologique décennal du Centre Nucléaire de Production d'Électricité du Tricastin (2001), Rapport IRSN/DEI/SESURE n 06-05, $120 \mathrm{p}$.

Gontier G., Grenz C., Calmet D., Sacher M. (1992) The contribution of Mytilus sp. in radionuclide transfer between water comlumn and sediments in the estuarine and delta systems of the Rhône River, Estuarine, Coastal and Shelf Science 34, 593-601.

Guénot J. (1984) Comportement du tritium dans les végétaux supérieurs, Rapport CEA-R-5269, 50 p.

IAEA (2010) Chapter 12: Specific activity models and parameter values for tritium, $14 \mathrm{C}$ and $36 \mathrm{Cl}$. In: Handbook of parameter values for the prediction of radionuclide transfer in terrestrial and freshwater environments. Vienna, Technical Report Series 472.

Jean-Baptiste P., Baumier D., Fourré E., Dapoigny A., Clavel B. (2007) The distribution of tritium in the terrestrial and aquatic environments of the Creys-Malville nuclear power plant (2002-2005), J. Environ. Rad. 94, 107-118.

Jean-Baptiste P., Fourré E., Dapoigny A., Baumier D., Baglan N., Alanic G. (2010) ${ }^{3} \mathrm{He}$ mass spectrometry for very low-level measurement of organic tritium in environmental samples, $J$. Environ. Rad. 101, 185-190.

Jeanmaire L., Vernois Y., Bullier D. (1973) Techniques de mesures utilisées pour l'étude du métabolisme du tritium organique, CEA Fontenay-aux-Roses, Note CEA-N-1652, 22 p.

Kirchmann R., Dupont J.C. (1981) Rôle du tritium dans les rejets d'effluents radioactifs liquides provenant d'installations nucléaires, Bull. Rech. Agron. Gembloux 16, 111-136.

Kirchmann R., Bonotto S., Soman S.D., Krishnamoorthy T.M., Iyengar T.S., Moghissi A.A. (1979) Transfer and incorporation of tritium in aquatic organisms. In: Proceedings of the international symposium on the behaviour of tritium in the environment, 16-20 October 1978, San Francisco, IAEA, pp. 187-204.

Le Guen B. (2008) Impact du tritium autour des centrales nucléaires EDF, Radioprotection 43, 177 191.

Leprieur F. (1999) Bilan radioécologique décennal de l'environnement terrestre et aquatique du Centre Nucléaire de Production d'Électricité de Belleville-sur-Loire (1998), IPSN/DPRE/SERNAT. Document SERNAT 99-012, 77 p. et annexes.

Leprieur F., Gontier G. (2001) Deuxième bilan radioécologique décennal du Centre Nucléaire de Production d'Électricité du Bugey (1999), Rapport DPRE/SERNAT 2001-29, 146 p.

McCubbin D., Leonard K.S., Bailey T.A., Williams J., Tossell P. (2001) Incorporation of Organic Tritium $\left({ }^{3} \mathrm{H}\right)$ by Marine Organisms and Sediment in the Severn Estuary/Bristol Channel (UK), Marine Pollution Bulletin 42, 852-863.

MEDD/HYDRO (2010) Banque Hydro - http://www.hydro.eaufrance.fr/.

Murith C., Zeller W., Hammans M., Zeller A. (2006) Réexamen de la justification du tritium dans l'industrie horlogère, Radioprotection 41, 189-200.

Murphy C.E. Jr., Sweet C.W., Fallon R.D. (1982) Tritium transport around nuclear facilities, Nuclear Safety 23, 677-685.

OFSP (2010) Radioactivité de l'environnement et doses de rayonnements en Suisse, Bern, Office Fédéral de la Santé Publique. 
Pally M., Barré A., Foulquier L. (1993) Tritium associé à la matière organique de sédiments, végétaux et poissons des principaux cours d'eau français, Verh. Internat. Verein. Limnol. 25, 285-289.

Parache V. (2009) Bilan radioécologique décennal de l'environnement proche du Centre Nucléaire de Production d'Électricité de Saint-Alban-Saint-Maurice-l'Exil (2005), Rapport IRSN/DEI/SESURE n 2009-08, 150 p.

Parache V. (2010a) Bilan radioécologique décennal de l'environnement proche du Centre Nucléaire de Production d'Électricité de Chooz (2006), Rapport IRSN/DEI/SESURE 2010-15, 156 p.

Parache V. (2010b) Bilan radioécologique décennal de l'environnement proche du Centre Nucléaire de Production d'Électricité de Nogent-sur-Seine (2008), Rapport IRSN/DEI/SESURE n 2010 , $146 \mathrm{p}$.

Siclet F. (2001) Transfert jusqu'a l'estuaire des radionucléides rejetés par les CNPE du bassin de la Loire, Hydroécol. Appl. 13, 43-83.

Smeesters P., Masse R. (2010) Groupe de réflexion tritium : synthèse des travaux et recommandations. In: Livre blanc du tritium, ASN, Paris, pp. 6-12.

SUBATECH (2010) Suivi radioécologique de l'environnement des C.N.P.E. du bassin de la Garonne Année 2009, Rapport SUB/RE/RC/O-G.

Workman W.J.G., Kim S.B., Kotzer T.G. (2005) Interlaboratory comparison of organically bound tritium measurements in environmental Samples, Fusion Science and Technology 48, 763-766. 